
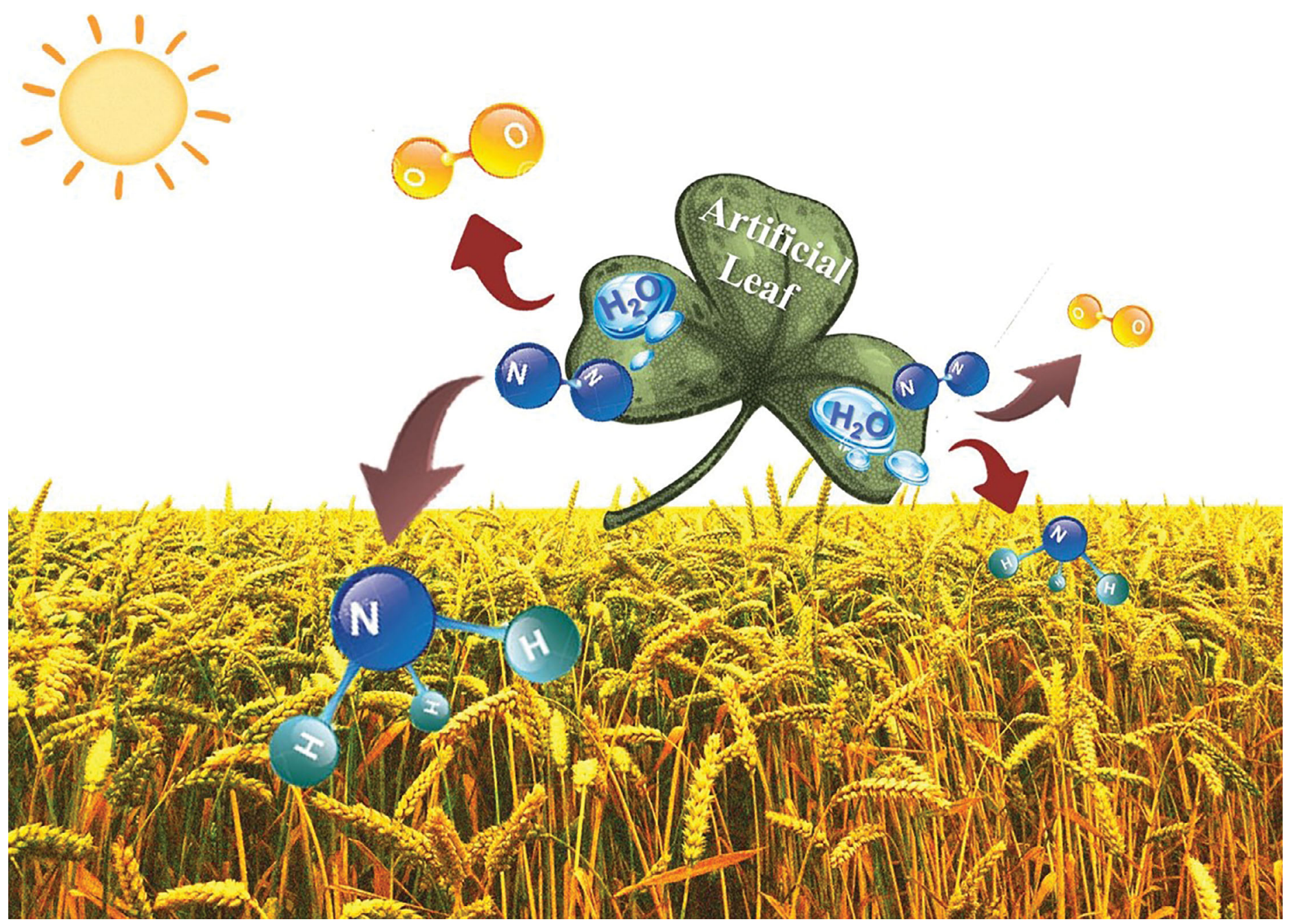

Showcasing research from Professor Junwang Tang's laboratory, Department of Chemical Engineering, University College London, London, United Kingdom.

Progress and challenges in photocatalytic ammonia synthesis

The group of Solar Energy and Advanced Materials, led by Prof. Junwang Tang focuses on photocatalytic small molecule activation (including $\mathrm{CH}_{4}, \mathrm{~N}_{2}, \mathrm{H}_{2} \mathrm{O}, \mathrm{C}_{6} \mathrm{H}_{6}$ and $\mathrm{CO}_{2}$ ) to high-value chemicals/fuels and microwave catalysis (e.g. plastic chemical recycling), together with microwave-intensified chemical processes. In parallel, the group has state-of-the-art spectroscopies to investigate the underlying charge dynamics and kinetics. This review puts forward a few critical issues existing in nitrogen photoreduction, along with a comprehensive discussion of strategies for improvement of $\mathrm{NH}_{3}$ yield, aiming to make substantial contribution towards advancing this field.

As featured in:

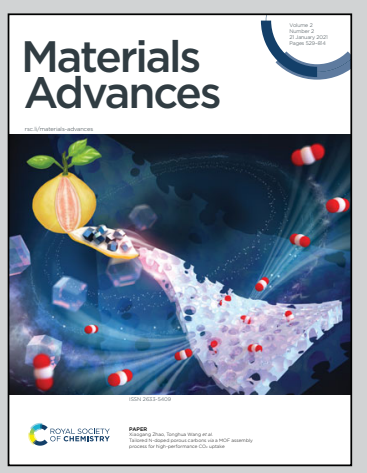

See Junwang Tang et al., Mater. Adv., 2021, 2, 564. 


\section{D) Check for updates}

Cite this: Mater. Adv., 2021, 2, 564

Received 10th August 2020, Accepted 1st November 2020 DOI: $10.1039 / \mathrm{d} 0 \mathrm{ma} 00590 \mathrm{~h}$

rsc.li/materials-advances

\title{
Progress and challenges in photocatalytic ammonia synthesis
}

\author{
Qing Han, (D) $\dagger^{\mathrm{ab}}$ Haimiao Jiao, (D) $\dagger^{\mathrm{b}}$ Lunqiao Xiong (D) ${ }^{\mathrm{b}}$ and Junwang Tang (D) $\star^{\mathrm{b}}$
}

\begin{abstract}
Photocatalytic ammonia $\left(\mathrm{NH}_{3}\right)$ synthesis from $\mathrm{N}_{2}$ and water driven by solar energy is a sustainable and environmentally friendly technology, which has gained considerable attention in recent years. In this review, the recent development in the fundamental understanding of photocatalytic $\mathrm{NH}_{3}$ synthesis and the methods of precise $\mathrm{NH}_{3}$ detection are summarized. More importantly the strategy for surface engineering and interface engineering of photocatalysts toward photocatalytic $\mathrm{NH}_{3}$ production has been thoroughly analyzed with the aim to stimulate critical thinking about the effective methodology for catalyst modification instead of exploring new materials. At the end the challenges and a few concerns are raised from the current reports and future perspectives in this research field are discussed targeting to clarify the reliability and reproducibility of the photochemical process and to direct the future research direction, such as flow reactor design and in-depth understanding of the underlying reaction pathway.
\end{abstract}

\section{Introduction}

Ammonia $\left(\mathrm{NH}_{3}\right)$ has been regarded as one of the most important chemical products as feedstock for fertilizers and for various chemicals. ${ }^{1,2}$ The production of $\mathrm{NH}_{3}$ plays a prominent role in global economy with an annual yield of more than 200 million tons. ${ }^{3}$ The industrial synthesis of $\mathrm{NH}_{3}$ is dominated

\footnotetext{
${ }^{a}$ Key Laboratory of Cluster Science, Ministry of Education of China, Key Laboratory of Photoelectronic/Electrophotonic Conversion Materials, School of Chemistry and Chemical Engineering, Beijing Institute of Technology, Beijing 100081, China

${ }^{b}$ Department of Chemical Engineering, University College London,

London WC1E 7JE, UK. E-mail: junwang.tang@ucl.ac.uk

$\dagger$ Co-first authors: These authors contributed equally to this work.
}

by the revolutionary Haber-Bosch process, in which pure $\mathrm{H}_{2}$ and $\mathrm{N}_{2}$ are reacted under high pressure at high temperature over Fe-based catalysts, which consumes 1-2\% of total global fossil fuels and releases around 300 million tons of planetwarming $\mathrm{CO}_{2}$ into the atmosphere annually. ${ }^{4-7}$ The development of environmentally friendly, sustainable strategies with high efficiency of $\mathrm{NH}_{3}$ production under mild conditions is highly desirable but challenging.

Currently, there are a few green routes reported for ammonia synthesis. One is biological nitrogen fixation which mostly relies on diazotrophs in nature. ${ }^{8}$ Another one is electrochemical reduction of $\mathrm{N}_{2}$, which uses electricity to produce $\mathrm{NH}_{3} \cdot{ }^{9,10}$ Compared with the stringent conditions of biological nitrogen fixation and the requirement of highly conductive electrolytes

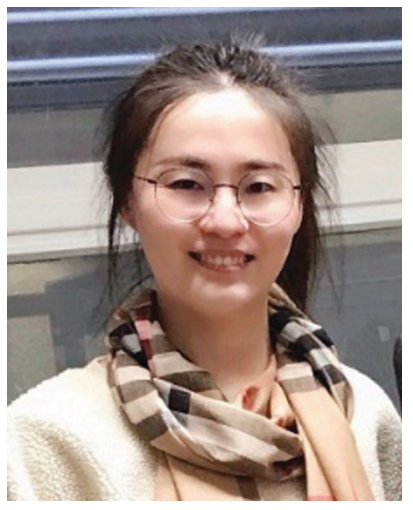

Qing Han
Qing Han is an Assistant Professor of Chemistry at Beijing Institute of Technology (Beijing, China). She received her $P h D$ in chemistry from Beijing Institute of Technology. Her research focuses on (i) the design and synthesis of nanocatalysts for photocatalysis and electrocatalysis, and (ii) device fabrication of carbonbased nanomaterials for energy conversion.

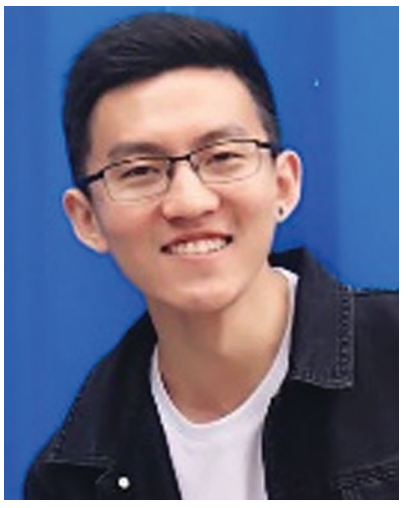

Haimiao Jiao received his BEng and MSc in Chemical Engineering from Tianjin University and Imperial College London, respectively. Currently, he is a PhD student in Prof. Junwang Tang's group at the Department of Chemical Engineering, University College London. His research interests focus on photocatalytic hydrogen production from methanol and nitrogen reduction. 
and costly electrodes used in electrochemical reduction of $\mathrm{N}_{2}$, photocatalytic ammonia synthesis using sustainable solar energy operated under either aqueous or gaseous conditions is rather different, maybe also economical, from the other methods. If the efficiency of solar driven $\mathrm{NH}_{3}$ synthesis could be dramatically improved, this method has strong potential to replace the current Haber-Bosch process. Photocatalytic reduction of $\mathrm{N}_{2}$ to produce $\mathrm{NH}_{3}$ by utilizing photocatalysts and water, driven by renewable solar energy, has huge potential, and thereby is very significant. ${ }^{11-17}$ Since the seminal work of Schrauzer and Guth in $1977,{ }^{17}$ in which the potential of the $\mathrm{TiO}_{2}$ photocatalyst for $\mathrm{N}_{2}$ reduction with water and $\mathrm{N}_{2}$ was revealed, various semiconductors have been studied for the photocatalytic production of $\mathrm{NH}_{3}$, such as $\mathrm{ZnO}, \mathrm{Fe}_{2} \mathrm{O}_{3}, \mathrm{Ga}_{2} \mathrm{O}_{3}$, $\mathrm{W}_{18} \mathrm{O}_{49}$, BiOBr, BiOCl, CdS, layered double hydroxides, polymeric carbon nitride and so on. ${ }^{18-25}$ The main challenges for the photocatalytic synthesis of $\mathrm{NH}_{3}$ are the chemical adsorption and activation of $\mathrm{N}_{2}$. To boost the efficiency of $\mathrm{NH}_{3}$ photosynthesis, the exploitation of highly active photocatalytic materials is crucial. Through surface/interface engineering of various photocatalysts, the photocatalytic reaction pathways can be modulated, therefore enhancing the $\mathrm{N}_{2}$ reduction reaction activity.

Complementary to other reviews in which different photocatalysts are summarized, ${ }^{6,13,18,19,22}$ this crucial review tries to avoid simply listing a large population of materials and mainly concentrates on recent advances and understanding in two fast moving areas, i.e. surface engineering and interface engineering, which have been proved to be efficient strategies to improve most photocatalysts' activity for $\mathrm{NH}_{3}$ synthesis, including defect engineering, morphology engineering, cocatalyst loading, and junction structure. The materials stated here are representative and highly efficient. Thus, this review aims at providing a comprehensive understanding of the rational design of highly efficient photocatalysts for $\mathrm{N}_{2}$ photoreduction. Apart from this, the recent understanding of the photocatalytic mechanism of $\mathrm{NH}_{3}$ synthesis and the methods to precisely evaluate the catalytic performance of a photocatalyst in $\mathrm{NH}_{3}$ synthesis will be discussed. Future research directions for further development of the photocatalytic synthesis of $\mathrm{NH}_{3}$ and some concerns will be highlighted at the end.

\section{Photocatalytic mechanism of $\mathrm{NH}_{3}$ synthesis}

By mimicking the natural process of photosynthesis, researchers intend to convert solar energy, $\mathrm{N}_{2}$ and water to $\mathrm{NH}_{3}$ by using photocatalysts. The overall reaction is listed as follows:

$$
2 \mathrm{~N}_{2}+6 \mathrm{H}_{2} \mathrm{O} \rightarrow 4 \mathrm{NH}_{3}+3 \mathrm{O}_{2}
$$

In this artificial photosynthesis, there are two interlinked conversion processes, water splitting and $\mathrm{N}_{2}$ reduction reaction (NRR). ${ }^{26}$ As shown in Fig. 1a, upon light irradiation, photoinduced electrons generated from photocatalysts populate the conduction band, leaving holes in the valence band. Subsequently, the escaped electrons diffuse across to the reactive sites on the surface of photocatalysts. The adsorbed $\mathrm{N}_{2}$ and $\mathrm{H}_{2} \mathrm{O}$ are catalyzed by these electrons and holes into $\mathrm{NH}_{3}$ and $\mathrm{O}_{2}$, respectively, along with the generation of the byproduct $\mathrm{H}_{2}$. Thus the key point for this process is not only to design a highly active photocatalyst but also to achieve high selectivity to $\mathrm{NH}_{3}$ instead of $\mathrm{H}_{2}$ gas as indicated in Fig. 1a.

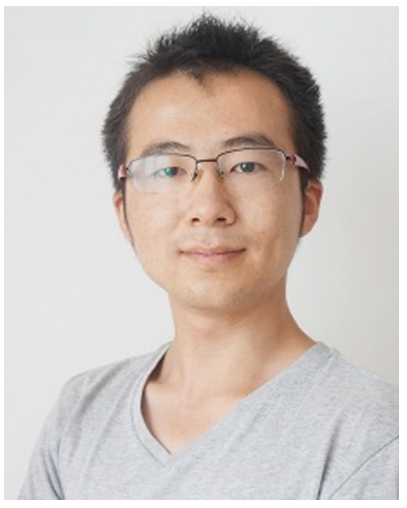

Lunqiao Xiong received his BSc and MEng in Materials Science from Xi'an Jiaotong University and Tsinghua University, respectively. At present, he is a PhD student in Prof. Junwang Tang's group at the UCL Department of Chemical Engineering. His current research focuses on photocatalytic selective oxidation.

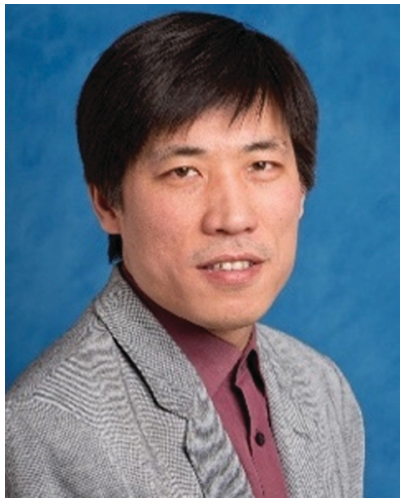

Junwang Tang
Junwang Tang is a Fellow of European Academy of Sciences, Fellow of the Royal Society of Chemistry and Professor of Materials Chemistry and Engineering in the Department of Chemical Engineering at University College London. His research interests encompass photocatalytic small molecule activation (e.g. $\mathrm{CH}_{4}, \mathrm{~N}_{2}, \mathrm{H}_{2} \mathrm{O}$, $\mathrm{CO}_{2}$ and $\left.\mathrm{C}_{6} \mathrm{H}_{6}\right)$ and microwave catalysis (plastic chemical recycling), together with microwave-intensified chemical processes. He has also received many awards, the latest of which is the IChemE Business Start-Up Award 2019. He also sits on the editorial/advisory board of several international journals, e.g. the Editor of Applied Catalysis B, Editor-in-Chief of Journal of Advanced Chemical Engineering, and Associate Editor of Chin. J. Catal. and Asia-Pacific Journal of Chemical Engineering. 
(b)

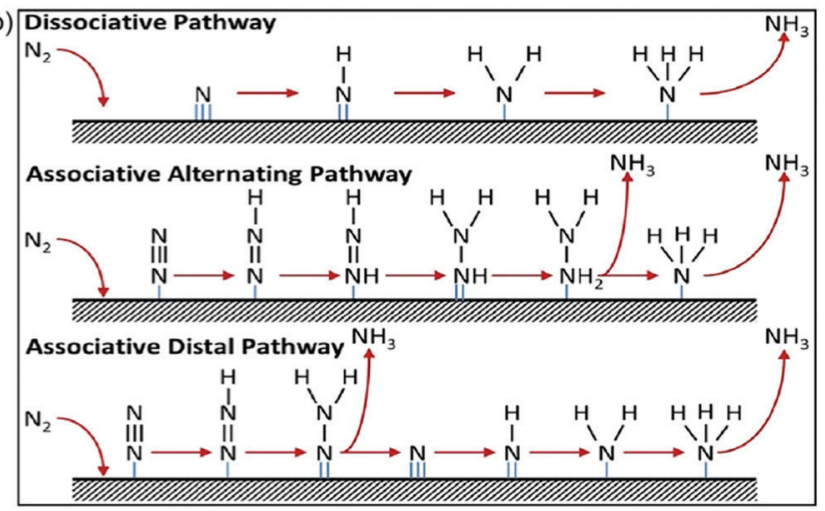

(a)

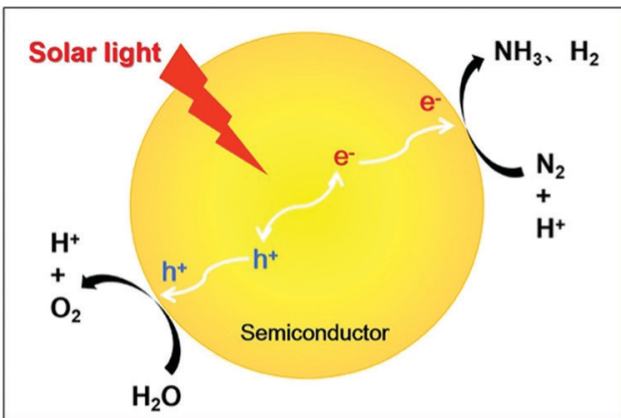

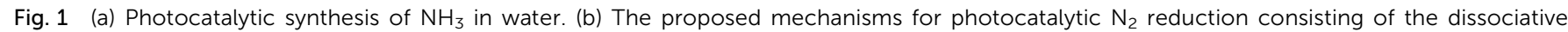

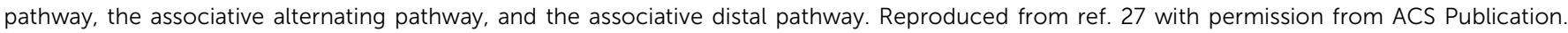

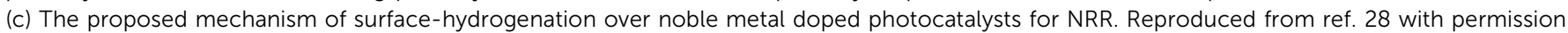
from ACS Publication.

Up to now, the mechanisms for $\mathrm{N}_{2}$ reduction to $\mathrm{NH}_{3}$ on photocatalysts have been divided into two categories based on the surface adsorption characteristics of catalysts: $N$-hydrogenation (Fig. 1b) and surface-hydrogenation (Fig. 1c). ${ }^{27,28}$ For the $N$-hydrogenation mechanism, there are two different pathways for NRR, dissociative and associative pathways. In the dissociative pathway, the $\mathrm{N}_{2}$ triple bond is broken before hydrogenation, followed by hydrogenation of the $\mathrm{N}$-adatoms into $\mathrm{NH}_{3}$ on the catalyst surface. The Haber-Bosch process is proved to be the dissociative pathway, which requires prohibitively high energy to cleave the $\mathrm{N} \equiv \mathrm{N}$ bond. Compared with the dissociative pathway, the associative pathway without the breaking of the $\mathrm{N} \equiv \mathrm{N}$ bond is generally used and accepted far more in the photocatalytic NRR. The nitrogen surface adsorption configuration determines the associative pathways for NRR; as shown in Fig. $1 b$, the hydrogenation of the adsorbed $N_{2}$ preferentially occurs at the terminal-site $\mathrm{N}$ owing to its relatively low steric hindrance for both the associative alternative pathway and the associative distal pathway. Having generated the first $\mathrm{NH}_{3}$, the other $\mathrm{N}$ begins a new hydrogenation circulation to produce a second $\mathrm{NH}_{3}$. It is actually impossible to identify the $\mathrm{N}_{2}$ configurations due to the fact that they are favored equally by the catalysts.

Recently, a novel surface-hydrogenation mechanism for NRR has been proposed (Fig. 1c), ${ }^{28}$ in which the adsorbed $\mathrm{H}^{+}$ is reduced into ${ }^{*} \mathrm{H}$ first, and then $\mathrm{N}_{2}$ molecules react with surface ${ }^{*} \mathrm{H}$ to generate ${ }^{*} \mathrm{~N}_{2} \mathrm{H}_{2}$, and finally the formed ${ }^{*} \mathrm{~N}_{2} \mathrm{H}_{2}$ intermediates are reduced into $\mathrm{NH}_{3}$. The first step is the trigger step, which enables the NRR to occur at a low potential. Furthermore, the second step is the rate determining step because it needs to conquer a high energy barrier $\left(E_{\mathrm{a}}\right)$ to break the $\mathrm{N} \equiv \mathrm{N}$ bond. The $E_{\mathrm{a}}$ of hydrogen evolution reaction is always lower than that of $\mathrm{NRR}$, and therefore the $\mathrm{NH}_{3}$ production rate is relatively low.

As a complete cycle indicated in Fig. 1a is the ideal process but very challenging, in most cases (or in the majority of the reported cases), a half reaction was investigated in which $\mathrm{N}_{2}$ was reduced to $\mathrm{NH}_{3}$ in the presence of an efficient hole scavenger which was oxidized other than water oxidation. This half reaction is relatively easy and can be used to preliminarily scan different photocatalysts for $\mathrm{N}_{2}$ reduction. Bear in mind that this half reaction has advantages but does not indicate whether the photocatalyst selected is good for a complete cycle as indicated in Fig. 1a. The subsequent discussion will underline this in order to remind the audience of the large difference between a half reaction and the complete cycle.

\section{Determination of $\mathrm{NH}_{3}$}

$\mathrm{NH}_{3}$ detection is a necessary subject for the photocatalytic synthesis of $\mathrm{NH}_{3}$, which is of great significance in the design of high performance photocatalysts. There are four common methods of $\mathrm{NH}_{3}$ analysis such as colorimetric assays, ${ }^{15} \mathrm{~N}$ 
(a)

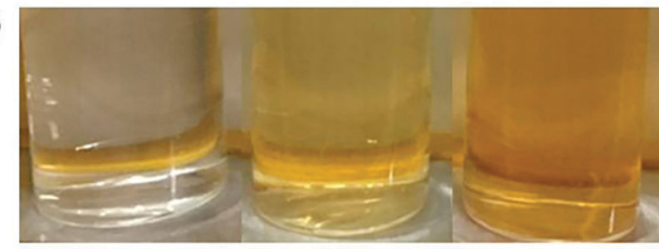

(b)

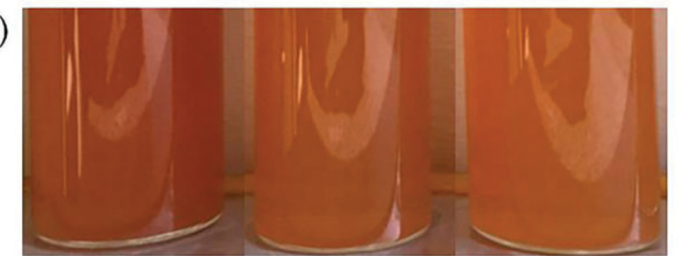

(d)

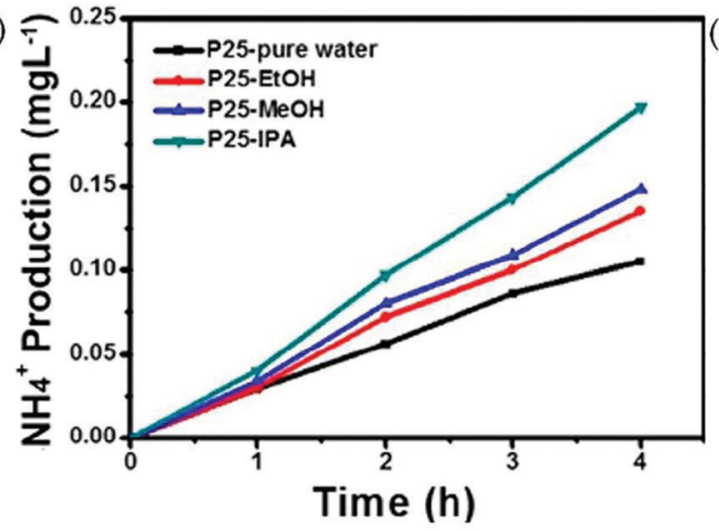

(c)

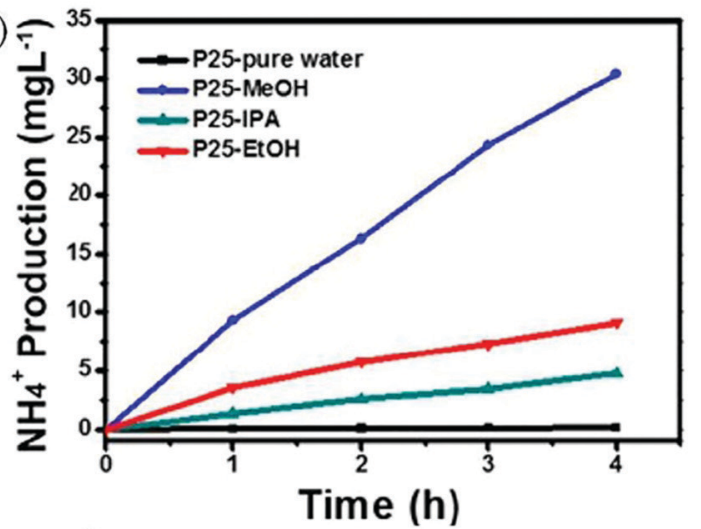

(e)

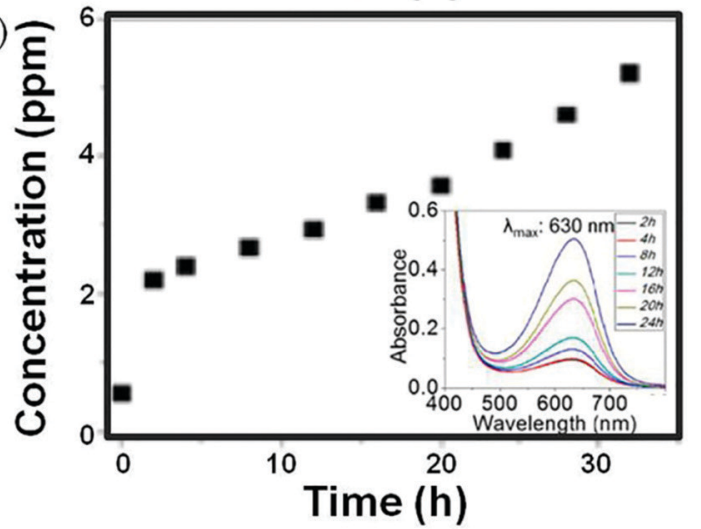

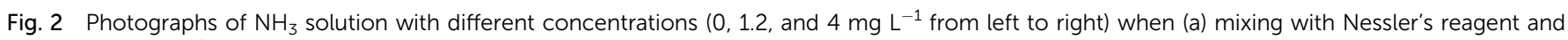

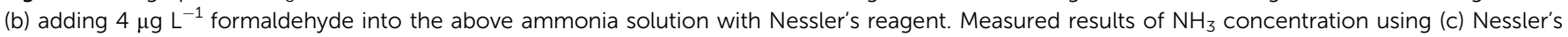

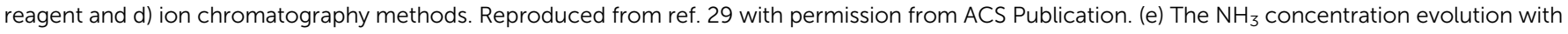

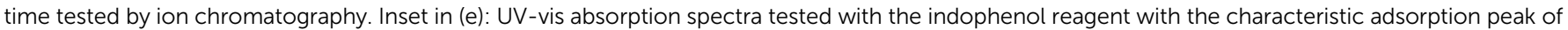
$630 \mathrm{~nm}$ wavelength. Reproduced from ref. 30 with permission from ACS Publication.

isotope labeling, ion chromatography, and in situ infrared spectroscopic measurements. ${ }^{12}$ The colorimetric assays include Nessler, phenate, and indophenol blue tests for $\mathrm{NH}_{3}$ detection. Among the colorimetric assays, Nessler's reagent has been widely used in $\mathrm{NH}_{3}$ detection due to its advantages of simplicity and low cost. ${ }^{29}$ However, it is reported that the sacrificial agents (i.e., alcohol) used in the photocatalytic synthesis of $\mathrm{NH}_{3}$ systems can be oxidized to carbonyl compounds, which will disturb $\mathrm{NH}_{3}$ detection in Nessler's reagent detection, thus yielding misleading results. Fig. 2a shows photographs of a solution containing Nessler's reagent and different concentrations of $\mathrm{NH}_{3}$ solutions $\left(0,1.2\right.$, and $\left.4 \mathrm{mg} \mathrm{L}^{-1}\right) .{ }^{29}$ It can be seen that the solution color is turning from light yellow to brown with the increase in the concentration of $\mathrm{NH}_{3}$. When $4 \mu \mathrm{g} \mathrm{L}^{-1}$ of formaldehyde is added, all the above solutions change into brown, indicating that trace formaldehyde can cause a remarkable color change (Fig. 2b). Therefore, the method of Nessler's reagent is not able to detect $\mathrm{NH}_{3}$ production in the presence of this or similar sacrificial agents. In contrast, the generated carbonyl compounds have no effect on the testing method of ion chromatography (Fig. 2c and d). It is strongly recommended that the colorimetric assay methods have to be accompanied by other methods such as ion chromatography, ${ }^{15} \mathrm{~N}$ isotope labeling, and in situ infrared spectroscopic measurements, to avoid such misleading results. Fig. 2e shows the photocatalytic performance of $\mathrm{NH}_{3}$ synthesis via ion chromatography and the indophenol test. ${ }^{30}$ The UV-vis spectra of the reaction solution containing $\mathrm{NH}_{3}$ exhibit a maximum absorption at $630 \mathrm{~nm}$ (inset in Fig. 2e), which is ascribed to the indophenols. The production of $\mathrm{NH}_{3}$ measured by ion chromatography increases with the reaction time (Fig. 2e), consistent with the results obtained by the indophenol test, indicating that indophenol is to some extent reliable.

Extensive studies on the determination of $\mathrm{NH}_{3}$ have revealed that the indophenol blue method, Nessler's reagent method, and the ion chromatography method are accurate when the concentration of $\mathrm{NH}_{3}$ is less than $500 \mu \mathrm{g} \mathrm{L} \mathrm{L}^{-1}$, but the indophenol blue method tends to be less accurate at higher concentrations or under acidic conditions. Besides, the presence of carbonyl compounds from scavengers will enhance light adsorption in Nessler's reagent method, thus leading to an interfered detection of $\mathrm{NH}_{3}$. Thus, to guarantee accuracy and reliability of $\mathrm{NH}_{3}$ quantification, at least two detection methods such as the ion chromatography method and Nessler's reagent method are recommended. Furthermore, because of trace $\mathrm{NH}_{3}$ in the ambient atmosphere, it is necessary to test the isotopically labeled ${ }^{15} \mathrm{~N}_{2}$ to confirm the source of nitrogen in the product. 


\section{Strategies to improve photocatalytic efficiency}

As mentioned above there are a few reviews summarizing the activity of diverse photocatalysts. ${ }^{6,13,18,19,22}$ This critical review will not overlap with these reviews, but will only concentrate on the efficient strategies to improve (i) $\mathrm{N}_{2}$ adsorption and (ii) charge separation. Such strategies include surface engineering and interface engineering for $\mathrm{NH}_{3}$ synthesis. The photocatalytic process of $\mathrm{NH}_{3}$ production generally includes four steps, photogeneration of charge carriers followed by their migration to the active sites, $\mathrm{N}_{2}$ chemisorption, surface photo-oxidation and photo-reduction, and the desorption $\mathrm{NH}_{3}$ from photocatalysts. Following the above key steps, photocatalysts can be engineered to greatly promote their catalytic activities toward $\mathrm{NH}_{3}$ synthesis. As steps (ii), (iii) and (iv) all take place on the surface, through the study of surface regulations and interface modulations on the photocatalysts, their photocatalytic performance can be maneuvered. The surface regulations, including defect engineering and morphology engineering, significantly determine the photocatalytic activity because they can promote the surface adsorption and activation of $\mathrm{N}_{2}{ }^{23,31-40,54-57,64-71}$ The interface modulations, including modification with cocatalysts and semiconductors, greatly affect the charge transfer and separation efficiency. ${ }^{89-101,109-111}$ We will discuss these factors one by one below. In order to provide an intuitively systematic comparison of photocatalytic $\mathrm{N}_{2}$ reduction systems, we list photocatalysts, reactants, $\mathrm{NH}_{3}$ production rate, in particular the apparent quantum efficiency and detection method as shown in Table 1.

\section{Surface regulations}

Defect engineering. It is widely reported that vacancies in a photocatalyst can improve $\mathrm{N}_{2}$ adsorption, to some extent even charge separation. There are different populations of vacancies, including oxygen vacancies, nitrogen vacancies, carbon vacancies, as well as sulfur vacancies.

Oxygen vacancies. The introduction of oxygen vacancies (OVs) can effectively facilitate $\mathrm{N}_{2}$ adsorption and activation, as well as induce the generation of mid-gap states to promote the separation of photogenerated charge carriers. ${ }^{31-42}$ For instance, Zhang et al. reported that BiOBr nanosheets with OVs on the exposed $\{001\}$ facets (BOB-001-OV) could stretch the $\mathrm{N} \equiv \mathrm{N}$ bonds from $1.078 \AA$ to $1.133 \AA$, which gave strong evidence for $\mathrm{N}_{2}$ activation over the OVs. ${ }^{31}$ Theoretical calculations showed that the OV induced defect states could serve as the electron acceptor (Fig. 3a) to effectively suppress the recombination of electrons/holes and enhance the charge transfer from BOB-001-OV to the $\mathrm{N}_{2}$ molecule. Fluorescence spectroscopy showed that the average lifetime $(\tau)$ of BOB-001-OV was $2.15 \mathrm{~ns}$, around two times higher than that of BiOBr without OVs (BOB-001-H, Fig. 3b), proving that the OVs could promote the migration of charge carriers. With the function of OVs, BOB001-OV showed a $\mathrm{NH}_{3}$ yield rate of $104.3 \mu \mathrm{mol} \mathrm{g}^{-1} \mathrm{~h}^{-1}$ under visible light illumination in the absence of organic scavengers and noble-metal cocatalysts (Fig. 3c), and an apparent quantum efficiency (AQE) of $0.23 \%$ at $420 \mathrm{~nm}$. Meanwhile, the amount of generated $\mathrm{O}_{2}$ was stoichiometrically approaching $3 / 4$ of the produced $\mathrm{NH}_{3}$, which proved that water could act as an electron donor to achieve a complete chemical cycle in $\mathrm{NH}_{3}$ photosynthesis.

Apart from the research on bismuth oxyhalide materials, Shiraishi's group utilized a series of commercial $\mathrm{TiO}_{2}$ to study the effect of OVs on the photoreduction of $\mathrm{N}_{2}$ to $\mathrm{NH}_{3}{ }^{32}$ A commercial $\mathrm{TiO}_{2}$ of JRC-TIO-6 showed an excellent performance with a steadily increasing $\mathrm{NH}_{3}$ production within $100 \mathrm{~h}$ under UV light irradiation in pure water. They proposed that the $\mathrm{Ti}^{3+}$ species formed at the OVs on the surface of $\mathrm{TiO}_{2}$ served as active sites, which could effectively trap the electrons and promote $\mathrm{N}_{2}$ dissociation, resulting in a photocatalytic $\mathrm{NH}_{3}$ production rate of $2.5 \mu \mathrm{mol} \mathrm{h}{ }^{-1}$.

In order to create the OVs, most studies used oxygencontaining catalysts. In recent years, layered double hydroxide (LDH, Fig. 4a) materials have attracted substantial attention due to their tunable electron structure and low cost. ${ }^{34}$ Zhang's group firstly used the CuCr-LDH nanosheets (CuCr-NS) with OVs as efficient photocatalysts for $\mathrm{NH}_{3}$ photosynthesis. ${ }^{34}$ The OVs over CuCr-NS surface were created by the reduction of thickness and the in-plane compressive strain (Fig. 4b). The optimal CuCr-NS exhibited a $\mathrm{NH}_{3}$ production rate of $78.6 \mu \mathrm{mol} \mathrm{g}{ }^{-1} \mathrm{~h}^{-1}$ under full-spectrum irradiation (Fig. 4c), and an AQE of $2.4 \%$ at $400 \mathrm{~nm}$. Subsequently, they further reported another work on ZnAl-LDH nanosheets for optimal OVs by incorporating coordinately unsaturated $\mathrm{Cu}^{\delta+}$ species. ${ }^{35}$ The DFT calculations implied that the OVs and $\mathrm{Cu}^{\delta+}$ species in ZnAl-LDH could effectively promote $\mathrm{N}_{2}$ adsorption and activation, leading to a remarkable $\mathrm{NH}_{3}$ yield of $110 \mu \mathrm{mol} \mathrm{L}^{-1} \mathrm{~h}^{-1}$ in pure water under UV-vis irradiation. Therefore, the creation of OVs on the surface of photocatalysts paves a way for developing other innovative materials toward photocatalytic $\mathrm{N}_{2}$ reduction.

Nitrogen vacancies/carbon vacancies. Inspired by the conclusion of $\mathrm{N}_{2}$ activation and efficient electron transfer by OVs on the catalyst surface, nitrogen vacancies (NVs) were later studied for the NRR process. Because of its excellent optical properties, low cost, and good stability, graphitic carbon nitride $\left(\mathrm{g}-\mathrm{C}_{3} \mathrm{~N}_{4}\right)$ has attracted much scientific interest in the field of photocatalysis. ${ }^{42-53}$ Dong et al. have reported the application of NV-incorporated g- $\mathrm{C}_{3} \mathrm{~N}_{4}\left(\mathrm{~V}-\mathrm{g}-\mathrm{C}_{3} \mathrm{~N}_{4}\right)$ for $\mathrm{N}_{2}$ photoreduction. ${ }^{54}$ They found that the $\mathrm{N}_{2}$ adsorption amount of $\mathrm{V}-\mathrm{g}-\mathrm{C}_{3} \mathrm{~N}_{4}$ was $75.1 \mathrm{~cm}^{3} \mathrm{~g}^{-1}, 2.4$ times higher than that of $\mathrm{g}-\mathrm{C}_{3} \mathrm{~N}_{4}$ without NVs $\left(30.9 \mathrm{~cm}^{3} \mathrm{~g}^{-1}\right)$, which suggested that the NVs on the surface of $\mathrm{V}$-g- $\mathrm{C}_{3} \mathrm{~N}_{4}$ could provide a number of chemisorption and activation sites for $\mathrm{N}_{2}$ molecules, leading to an $\mathrm{NH}_{3}$ production rate of $1.24 \mathrm{mmol} \mathrm{h}^{-1} \mathrm{~g}_{\text {cat }}{ }^{-1}$ under visible light irradiation in the presence of methanol as a hole scavenger. Recently, a g$\mathrm{C}_{3} \mathrm{~N}_{4}$ catalyst modified with cyano groups $(-\mathrm{C} \equiv \mathrm{N})$ and intercalated $\mathrm{K}^{+}(\mathrm{mCNN})$ was prepared as an efficient photocatalyst for NRR. ${ }^{55}$ Relative to pristine g- $\mathrm{C}_{3} \mathrm{~N}_{4}$, the absorption spectrum of mCNN was extended to the whole visible region (Fig. 5a), resulting from the $\pi-\pi^{*}$ electronic transitions in the conjugated heterocyclic rings and the $n-\pi^{*}$ electronic transitions at the 
Table 1 Photocatalytic performance of different catalysts for $\mathrm{NH}_{3}$ synthesis

\begin{tabular}{|c|c|c|c|c|c|c|c|}
\hline Catalyst & $\begin{array}{l}\text { Reaction } \\
\text { medium }\end{array}$ & Scavenger & Light source & Ammonia yield & $\begin{array}{l}\text { Apparent quantum } \\
\text { efficiency (AQE) }\end{array}$ & $\begin{array}{l}\text { Ammonia detection } \\
\text { method }\end{array}$ & Ref. \\
\hline BiOBr-001-OV & $\mathrm{N}_{2} / \mathrm{H}_{2} \mathrm{O}(\mathrm{l})$ & None & $\lambda>420 \mathrm{~nm}$ & $104.3 \mu \mathrm{mol} \mathrm{g}^{-1} \mathrm{~h}^{-1}$ & $0.23 \%$ at $420 \mathrm{~nm}$ & Nessler's reagent & 31 \\
\hline JRC-TIO-6 & $\mathrm{N}_{2} / \mathrm{H}_{2} \mathrm{O}(\mathrm{l})$ & 2-PrOH & $\lambda>280 \mathrm{~nm}$ & $2.5 \mu \mathrm{mol} \mathrm{g}{ }^{-1} \mathrm{~h}^{-1}$ & Not reported & Indophenol blue method & 32 \\
\hline CuCr-LDH & $\mathrm{N}_{2} / \mathrm{H}_{2} \mathrm{O}(\mathrm{l})$ & None & $\begin{array}{l}\text { Full } \\
\text { spectrum }\end{array}$ & $78.6 \mu \mathrm{mol} \mathrm{g}^{-1} \mathrm{~h}^{-1}$ & $2.4 \%$ at $400 \mathrm{~nm}$ & Nessler's reagent & 34 \\
\hline ZnAl-LDH & $\mathrm{N}_{2} / \mathrm{H}_{2} \mathrm{O}(\mathrm{l})$ & None & UV-vis & $110 \mu \mathrm{mol} \mathrm{L}{ }^{-1} \mathrm{~h}^{-1}$ & $\begin{array}{l}1.77 \% \text { at } 265 \mathrm{~nm}, \\
0.56 \% \text { at } 365 \mathrm{~nm}\end{array}$ & Ion chromatography & 35 \\
\hline $\mathrm{V}-\mathrm{g}-\mathrm{C}_{3} \mathrm{~N}_{4}$ & $\mathrm{~N}_{2} / \mathrm{H}_{2} \mathrm{O}(\mathrm{l})$ & Methanol & $\lambda>420 \mathrm{~nm}$ & $1.24 \mathrm{mmol} \mathrm{h}^{-1} \mathrm{~g}_{\text {cat }}{ }^{-1}$ & Not reported & Nessler's reagent & 54 \\
\hline mCNN & $\begin{array}{l}\mathrm{N}_{2} / \text { ethylene } \\
\text { glycol (l) }\end{array}$ & $\begin{array}{l}\text { Ethylene } \\
\text { glycol }\end{array}$ & $\lambda>400 \mathrm{~nm}$ & $3.42 \mathrm{mmol} \mathrm{g}^{-1} \mathrm{~h}^{-1}$ & Not reported & Nessler's reagent & 55 \\
\hline SCNNSs & $\mathrm{N}_{2} / \mathrm{H}_{2} \mathrm{O}(\mathrm{l})$ & Methanol & $\begin{array}{l}\text { Full } \\
\text { spectrum }\end{array}$ & $5.99 \mathrm{mmol} \mathrm{h}^{-1} \mathrm{~g}_{\text {cat }}{ }^{-1}$ & Not reported & Nessler's reagent & 56 \\
\hline $\mathrm{Mo}_{0.1} \mathrm{Ni}_{0.1} \mathrm{Cd}_{0.8} \mathrm{~S}$ & $\mathrm{~N}_{2} / \mathrm{H}_{2} \mathrm{O}(\mathrm{l})$ & Ethanol & UV & $3.2 \mathrm{mg} \mathrm{h}^{-1} \mathrm{~g}_{\text {cat }}^{-1}$ & Not reported & Nessler's reagent & 57 \\
\hline $\begin{array}{l}0.2 \mathrm{wt} \% \text { Fe-doped } \\
\mathrm{TiO}_{2}\end{array}$ & $\mathrm{~N}_{2} / \mathrm{H}_{2} \mathrm{O}(\mathrm{g})$ & None & UV & $11.6 \mu \mathrm{mol} \mathrm{g}^{-1} \mathrm{~h}^{-1}$ & Not reported & Indophenol blue method & 17 \\
\hline $\begin{array}{l}0.4 \text { wt } \% \text { Co-doped } \\
\mathrm{TiO}_{2}\end{array}$ & $\mathrm{~N}_{2} / \mathrm{H}_{2} \mathrm{O}(\mathrm{l})$ & None & UV & $6.3 \mu \mathrm{mol} \mathrm{g}^{-1} \mathrm{~h}^{-1}$ & Not reported & Indophenol blue method & 17 \\
\hline $\begin{array}{l}0.4 \text { wt } \% \text { Cr-doped } \\
\mathrm{TiO}_{2}\end{array}$ & $\mathrm{~N}_{2} / \mathrm{H}_{2} \mathrm{O}(\mathrm{l})$ & None & UV & $0.37 \mu \mathrm{mol} \mathrm{g}^{-1} \mathrm{~h}^{-1}$ & Not reported & Indophenol blue method & 17 \\
\hline $\begin{array}{l}0.4 \text { wt } \% \mathrm{Mo}^{-} \\
\text {doped } \mathrm{TiO}_{2}\end{array}$ & $\mathrm{~N}_{2} / \mathrm{H}_{2} \mathrm{O}(\mathrm{l})$ & None & UV & $6.7 \mu \mathrm{mol} \mathrm{g}^{-1} \mathrm{~h}^{-1}$ & Not reported & Indophenol blue method & 17 \\
\hline Fe-Doped $\mathrm{TiO}_{2}$ & $\mathrm{~N}_{2} / \mathrm{H}_{2} \mathrm{O}(\mathrm{l})$ & Ethanol & $\lambda=254 \mathrm{~nm}$ & $1.2 \mathrm{mmol} \mathrm{L}^{-1}$ & Not reported & Nessler's reagent & 64 \\
\hline Fe-Doped $\mathrm{SrMoO}_{4}$ & $\mathrm{~N}_{2} / \mathrm{H}_{2} \mathrm{O}(\mathrm{l})$ & None & UV-vis & $93.1 \mu \mathrm{mol} \mathrm{g}{ }^{-1} \mathrm{~h}^{-1}$ & Not reported & $\begin{array}{l}\text { Nessler's reagent, indophenol } \\
\text { blue method }\end{array}$ & 65 \\
\hline Fe-Doped BiOCl & $\mathrm{N}_{2} / \mathrm{H}_{2} \mathrm{O}(\mathrm{l})$ & None & $\begin{array}{l}\text { Full } \\
\text { spectrum }\end{array}$ & $1.022 \mathrm{mmol} \mathrm{g}^{-1} \mathrm{~h}^{-1}$ & $1.8 \%$ at $420 \mathrm{~nm}$ & Indophenol blue method & 66 \\
\hline Mo-Doped $\mathrm{W}_{18} \mathrm{O}_{49}$ & $\mathrm{~N}_{2} / \mathrm{H}_{2} \mathrm{O}(\mathrm{l})$ & $\mathrm{Na}_{2} \mathrm{SO}_{3}$ & $\begin{array}{l}\text { Full } \\
\text { spectrum }\end{array}$ & $195.5 \mu \mathrm{mol} \mathrm{g}^{-1} \mathrm{~h}^{-1}$ & $0.33 \%$ at $400 \mathrm{~nm}$ & $\begin{array}{l}\text { Nessler's reagent, ion } \\
\text { chromatography }\end{array}$ & 67 \\
\hline $\mathrm{Cu}$-Doped $\mathrm{TiO}_{2}$ & $\mathrm{~N}_{2} / \mathrm{H}_{2} \mathrm{O}(\mathrm{l})$ & None & $\begin{array}{l}\text { Full } \\
\text { spectrum }\end{array}$ & $78.9 \mu \mathrm{mol} \mathrm{g}^{-1} \mathrm{~h}^{-1}$ & $\begin{array}{l}0.08 \% \text { at } 600 \mathrm{~nm} \text {, } \\
0.05 \% \text { at } 700 \mathrm{~nm}\end{array}$ & $\begin{array}{l}\text { Nessler's reagent, ion } \\
\text { chromatography }\end{array}$ & 68 \\
\hline $\mathrm{FePt} @ \mathrm{C}_{3} \mathrm{~N}_{4}$ & $\mathrm{~N}_{2} / \mathrm{H}_{2}$ & None & $\lambda>400 \mathrm{~nm}$ & $63 \mu \mathrm{g} \mathrm{h}{ }^{-1} \mathrm{~g}^{-1}$ & $\begin{array}{l}0.15 \% \text { between } \\
450 \text { and } 500 \mathrm{~nm}\end{array}$ & $\begin{array}{l}\text { Colorimetric method, ion } \\
\text { chromatography }\end{array}$ & 72 \\
\hline B-Doped $\mathrm{g}-\mathrm{C}_{3} \mathrm{~N}_{4}$ & $\mathrm{~N}_{2} / \mathrm{H}_{2} \mathrm{O}(\mathrm{l})$ & $\mathrm{Na}_{2} \mathrm{SO}_{3}$ & $\lambda>400 \mathrm{~nm}$ & $313.9 \mu \mathrm{mol} \mathrm{g}^{-1} \mathrm{~h}^{-1}$ & $0.64 \%$ at $420 \mathrm{~nm}$ & Nessler's reagent & 73 \\
\hline C-Doped $\mathrm{TiO}_{2}$ & $\mathrm{~N}_{2} / \mathrm{H}_{2} \mathrm{O}(\mathrm{l})$ & Methanol & $\lambda>420 \mathrm{~nm}$ & $109.3 \mu \mathrm{mol} \mathrm{g}^{-1} \mathrm{~h}^{-1}$ & $2.4 \%$ at $400 \mathrm{~nm}$ & Ion chromatography & \\
\hline $\mathrm{Bi}_{5} \mathrm{O}_{7} \mathrm{I}-001$ & $\mathrm{~N}_{2} / \mathrm{H}_{2} \mathrm{O}(\mathrm{l})$ & Methanol & $280-800 \mathrm{~nm}$ & $111.5 \mu \mathrm{mol} \mathrm{g}{ }^{-1} \mathrm{~h}^{-1}$ & $5.1 \%$ at $365 \mathrm{~nm}$ & Nessler's reagent & 77 \\
\hline BiOCl-010 & $\mathrm{N}_{2} / \mathrm{H}_{2} \mathrm{O}(\mathrm{l})$ & Methanol & $\begin{array}{l}\text { Full } \\
\text { spectrum }\end{array}$ & $92.4 \mu \mathrm{mol} \mathrm{g}^{-1} \mathrm{~h}^{-1}$ & $4.3 \%$ at $254 \mathrm{~nm}$ & Nessler's reagent & 78 \\
\hline $\mathrm{BiO}$ & $\mathrm{N}_{2} / \mathrm{H}_{2} \mathrm{O}(\mathrm{l})$ & None & $\begin{array}{l}\text { Full } \\
\text { spectrum }\end{array}$ & $1226 \mu \mathrm{mol} \mathrm{g}^{-1} \mathrm{~h}^{-1}$ & Not reported & Indophenol blue method & 86 \\
\hline $\mathrm{Bi}_{5} \mathrm{O}_{7} \mathrm{Br}-\mathrm{NT}$ & $\mathrm{N}_{2} / \mathrm{H}_{2} \mathrm{O}(\mathrm{l})$ & None & $\lambda>400 \mathrm{~nm}$ & $1.38 \mathrm{mmol} \mathrm{g}^{-1} \mathrm{~h}^{-1}$ & $2.3 \%$ at $420 \mathrm{~nm}$ & Nessler's reagent & 87 \\
\hline eBP NFs & $\mathrm{N}_{2} / \mathrm{H}_{2} \mathrm{O}(\mathrm{l})$ & $\begin{array}{l}\mathrm{Na}_{2} \mathrm{SO}_{3}, \mathrm{Na}_{2} \mathrm{~S} \\
9 \mathrm{H}_{2} \mathrm{O}\end{array}$ & $\lambda=420 \mathrm{~nm}$ & $2.37 \mathrm{mmol} \mathrm{g}^{-1} \mathrm{~h}^{-1}$ & Not reported & Nessler's reagent & 88 \\
\hline $\mathrm{SiO}_{2} / \mathrm{C}-\mathrm{RP}$ & $\mathrm{N}_{2} / \mathrm{H}_{2} \mathrm{O}(\mathrm{l})$ & None & $\begin{array}{l}\text { Full } \\
\text { spectrum }\end{array}$ & $0.73 \mu \mathrm{mol} \mathrm{h}^{-1}$ & Not reported & Ion chromatography & 89 \\
\hline Ru-Loaded $\mathrm{TiO}_{2}$ & $\mathrm{~N}_{2} / \mathrm{H}_{2} \mathrm{O}(\mathrm{l})$ & Methanol & $\begin{array}{l}\text { Full } \\
\text { spectrum }\end{array}$ & $29.4 \mu \mathrm{mol} \mathrm{g}^{-1} \mathrm{~h}^{-1}$ & Not reported & Indophenol blue method & 93 \\
\hline Ru-Loaded $\mathrm{TiO}_{2}$ & $\mathrm{~N}_{2} / \mathrm{H}_{2} \mathrm{O}(\mathrm{l})$ & Ethanol & $\begin{array}{l}\text { Full } \\
\text { spectrum }\end{array}$ & $56.3 \mu \mathrm{g} \mathrm{h}^{-1} \mathrm{~g}_{\text {cat }}{ }^{-1}$ & Not reported & Indophenol blue method & 95 \\
\hline $\mathrm{Au} / \mathrm{TiO}_{2}-\mathrm{OV}$ & $\mathrm{N}_{2} / \mathrm{H}_{2} \mathrm{O}(\mathrm{l})$ & Methanol & $\lambda>420 \mathrm{~nm}$ & $78.6 \mu \mathrm{mol} \mathrm{g}{ }^{-1} \mathrm{~h}^{-1}$ & $0.82 \%$ at $550 \mathrm{~nm}$ & Indophenol blue method & 96 \\
\hline $\mathrm{Ti}_{3} \mathrm{C}_{2}-\mathrm{P} 25$ & $\mathrm{~N}_{2} / \mathrm{H}_{2} \mathrm{O}(\mathrm{l})$ & None & $\begin{array}{l}\text { Full } \\
\text { spectrum }\end{array}$ & $10.74 \mu \mathrm{mol} \mathrm{g}^{-1}$ & Not reported & Ion chromatography & 103 \\
\hline Pt-Loaded ZnO & $\mathrm{N}_{2} / \mathrm{H}_{2} \mathrm{O}(\mathrm{l})$ & $\mathrm{Na}_{2} \mathrm{SO}_{3}$ & UV & $860 \mathrm{mmol} \mathrm{g}^{-1} \mathrm{~h}^{-1}$ & Not reported & Indophenol blue method & 104 \\
\hline SV-1T-MoS $2 / \mathrm{CdS}$ & $\mathrm{N}_{2} / \mathrm{H}_{2} \mathrm{O}(\mathrm{l})$ & Methanol & $420-780 \mathrm{~nm}$ & $\begin{array}{l}8220.83 \mu \mathrm{mol} \mathrm{L}^{-1} \\
\mathrm{~h}^{-1} \mathrm{~g}^{-1}\end{array}$ & $\begin{array}{l}4.424 \% \text { under } \\
\text { simulated solar } \\
\text { light }\end{array}$ & Nessler's reagent & 105 \\
\hline NiS/CdS & $\mathrm{N}_{2} / \mathrm{H}_{2} \mathrm{O}(\mathrm{l})$ & None & $\begin{array}{l}\text { Full } \\
\text { spectrum }\end{array}$ & $2.8 \mathrm{mg} \mathrm{L}^{-1} \mathrm{~h}^{-1}$ & $0.76 \%$ at $420 \mathrm{~nm}$ & Nessler's reagent & 106 \\
\hline Pt-SACs/CTF & $\mathrm{N}_{2} / \mathrm{H}_{2} \mathrm{O}(\mathrm{l})$ & None & $420-780 \mathrm{~nm}$ & $171.4 \mu \mathrm{mol} \mathrm{g}^{-1} \mathrm{~h}^{-1}$ & $1.4 \%$ at $420 \mathrm{~nm}$ & Nessler's reagent & 107 \\
\hline $\mathrm{Ru} / \mathrm{RuO}_{2} / \mathrm{g}-\mathrm{C}_{3} \mathrm{~N}_{4}$ & $\mathrm{~N}_{2} / \mathrm{H}_{2} \mathrm{O}(\mathrm{l})$ & Methanol & $\begin{array}{l}\text { Full } \\
\text { spectrum }\end{array}$ & $13.3 \mu \mathrm{mol} \mathrm{g}{ }^{-1} \mathrm{~h}^{-1}$ & Not reported & Ion chromatography & 108 \\
\hline Fe@graphene & $\mathrm{N}_{2} / \mathrm{H}_{2}, 200{ }^{\circ} \mathrm{C}$ & None & $200-600 \mathrm{~nm}$ & $420 \mu \mathrm{g} \mathrm{g}_{\text {cat }}^{-1} \mathrm{~h}^{-1}$ & Not reported & Indophenol blue method & 110 \\
\hline $\mathrm{g}-\mathrm{C}_{3} \mathrm{~N}_{4} / \mathrm{Bi}_{2} \mathrm{MoO}_{6}$ & $\mathrm{~N}_{2} / \mathrm{H}_{2} \mathrm{O}(\mathrm{l})$ & Methanol & $\lambda>420 \mathrm{~nm}$ & $3271 \mu \mathrm{mol} \mathrm{L}^{-1} \mathrm{~g}^{-1}$ & Not reported & Nessler's reagent & 111 \\
\hline $\mathrm{TiO}_{2} @ \mathrm{C} / \mathrm{g}-\mathrm{C}_{3} \mathrm{~N}_{4}$ & $\mathrm{~N}_{2} / \mathrm{H}_{2} \mathrm{O}(\mathrm{l})$ & Methanol & $\lambda>420 \mathrm{~nm}$ & $250.6 \mu \mathrm{mol} \mathrm{g}^{-1} \mathrm{~h}^{-1}$ & $0.14 \%$ at $420 \mathrm{~nm}$ & Nessler's reagent & 112 \\
\hline $\begin{array}{l}\mathrm{Bi}_{2} \mathrm{MoO}_{6} / \mathrm{OV}- \\
\mathrm{BiOBr}\end{array}$ & $\mathrm{N}_{2} / \mathrm{H}_{2} \mathrm{O}(\mathrm{l})$ & None & $\begin{array}{l}\text { Full } \\
\text { spectrum }\end{array}$ & $81.0 \mu \mathrm{mol} \mathrm{g}{ }^{-1} \mathrm{~h}^{-1}$ & Not reported & Nessler's reagent & 116 \\
\hline
\end{tabular}

active $\mathrm{N}$ defect sites $(-\mathrm{C} \equiv \mathrm{N})$, which indicated that the introduction of the $\mathrm{N}$-defect of $-\mathrm{C} \equiv \mathrm{N}$ could significantly change the optical properties. Fig. 5b displays the band structures of mCNN and pristine $\mathrm{g}-\mathrm{C}_{3} \mathrm{~N}_{4}$ through the results of experiments. 


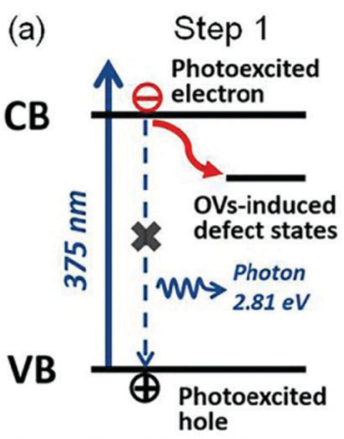

Step 1 and 2: OVs-induced defect states trap the excited electrons from $\mathrm{CB}$ of $\mathrm{BiOBr}$
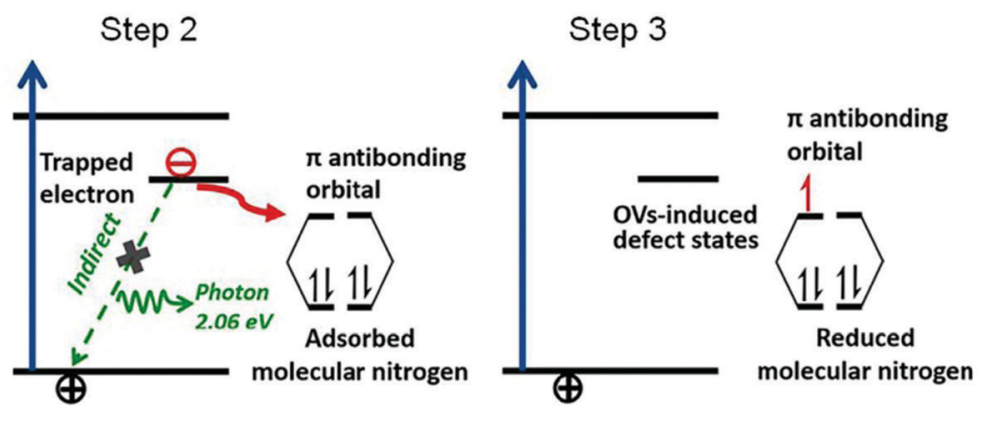

Step 3: The trapped electrons activate adsorbed $\mathrm{N}_{2}$ (b)

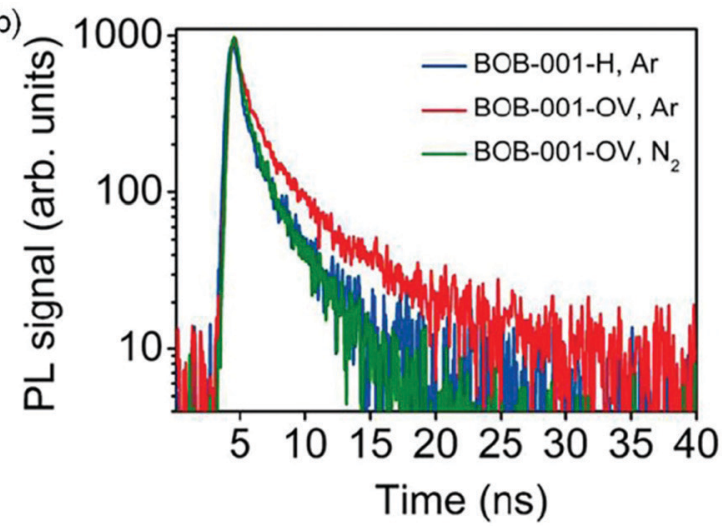

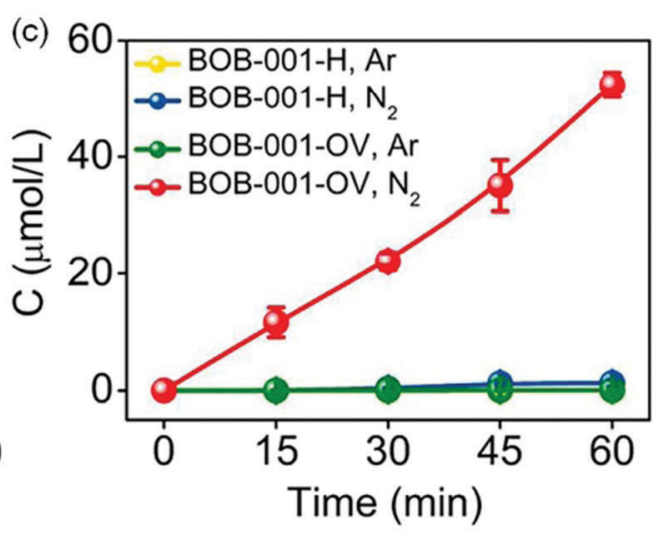

Fig. 3 Schematic of the OV-induced enhanced interfacial electron transfer processes over BOB-001-OV. (b) Time-resolved photoluminescence (PL) spectra of BOB-001-OV and BOB-001-H. (c) The photocatalytic $\mathrm{NH}_{3}$ production of BOB-001-OV and BOB-001- $\mathrm{H}$ under visible light $(\lambda>420 \mathrm{~nm})$. Reproduced from ref. 31 with permission from ACS Publication.

Compared with pristine $\mathrm{g}-\mathrm{C}_{3} \mathrm{~N}_{4}$, a subgap state was presented for mCNN, which could be ascribed to the $\mathrm{N}$ defect, resulting in a broad visible-light response. The enhanced EPR intensity (Fig. 5c) of mCNN compared to pristine $\mathrm{g}-\mathrm{C}_{3} \mathrm{~N}_{4}$ was assigned to the strong electron withdrawing groups of $-\mathrm{C} \equiv \mathrm{N}$ on mCNN, which could delocalize the isolated electrons in mCNN, therefore promoting the separation of photocarriers and increasing the generation of active radical species to boost the activity of $\mathrm{NH}_{3}$ photosynthesis. The rate of $\mathrm{NH}_{3}$ generation of the mCNN photocatalyst was measured to be $3.42 \mathrm{mmol} \mathrm{g}^{-1} \mathrm{~h}^{-1}$ with ethylene glycol as a scavenger under visible light irradiation (Fig. 5d), which was much higher than that of pristine g- $\mathrm{C}_{3} \mathrm{~N}_{4}$ $\left(1.11 \mathrm{mmol} \mathrm{g}^{-1} \mathrm{~h}^{-1}\right)$. The DFT calculations revealed that $\mathrm{K}^{+}$ could be linked to the unsaturated $\mathrm{C}$ centers by coordination as shown in Fig. 5e, which could adsorb $\mathrm{N}_{2}$ as the lone-pair electrons of nitrogen would fill the empty state in $\mathrm{K}^{+}(\Delta G=$ $-0.28 \mathrm{eV}$ ), followed by the rearrangement of $\mathrm{K}^{+}$with the $\mathrm{C}$ atom and the adsorption of $\mathrm{N}_{2}(\Delta G=-0.17 \mathrm{eV})$. Because of the large ion hydration free energy of $\mathrm{K}^{+}$, it could be extracted back into solution, then forming a $\mathrm{C}_{2} \mathrm{~N}_{4}$ ring $(\Delta G=1.64 \mathrm{eV})$ intermediate which could undergo a MvK process to regenerate $-\mathrm{C} \equiv \mathrm{N}$, thereby stabilizing the unsaturated $\mathrm{C}$ sites.

Apart from the above NVs on the $\mathrm{g}^{-} \mathrm{C}_{3} \mathrm{~N}_{4}$ photocatalyst, carbon vacancies (CVs) on a porous sulfur-doped $\mathrm{g}-\mathrm{C}_{3} \mathrm{~N}_{4}$ (SCNNSs) have also been exploited. ${ }^{56}$ The introduction of CVs was proved to play the same role as NVs, while the S-doping effect was demonstrated to improve the separation efficiency of photogenerated electron-hole pairs. Taking these advantages, the optimal SCNNSs showed an $\mathrm{NH}_{3}$ production rate of $5.99 \mathrm{mmol} \mathrm{h}^{-1} \mathrm{~g}_{\mathrm{cat}}{ }^{-1}$.

Sulfur vacancies. Considering the importance of the sulfur element in nitrogenases, a few have reported the effects of sulfur vacancies (SVs) on $\mathrm{N}_{2}$ photoreduction. It was reported that SVs could be created on the surface of $\mathrm{Mo}_{0.1} \mathrm{Ni}_{0.1} \mathrm{Cd}_{0.8} \mathrm{~S}$ by co-doping Mo and Ni into CdS. ${ }^{57} \mathrm{~N}_{2}$-temperature-programmed desorption ( $\left.\mathrm{N}_{2}-\mathrm{TPD}\right)$ results illustrated that the SVs on the surface of $\mathrm{Mo}_{0.1} \mathrm{Ni}_{0.1} \mathrm{Cd}_{0.8} \mathrm{~S}$ obviously enhanced $\mathrm{N}_{2}$ chemisorption (Fig. 6a), which was beneficial for NRR. Importantly, the photocatalytic $\mathrm{NH}_{3}$ generation rate of $\mathrm{Mo}_{0.1} \mathrm{Ni}_{0.1} \mathrm{Cd}_{0.8} \mathrm{~S}$ was linearly related to its SV concentration as shown in Fig. 6b. As the concentration of SVs increased, there was an increase in the photocatalytic $\mathrm{NH}_{3}$ production rate. This result suggested that the SVs played a vital role in the photoreduction of $\mathrm{N}_{2}$. The $\mathrm{Mo}_{0.1} \mathrm{Ni}_{0.1} \mathrm{Cd}_{0.8} \mathrm{~S}$ with the highest SV concentration showed the highest $\mathrm{NH}_{3}$ production rate of $3.2 \mathrm{mg} \mathrm{h}^{-1} \mathrm{~g}_{\text {cat }}{ }^{-1}$ (Fig. 6b).

Doping. Metal doping is a very promising strategy to change the electronic structure and surface property of photocatalysts to enhance the photocatalytic activity. ${ }^{58-63}$ In one report, 
(a)

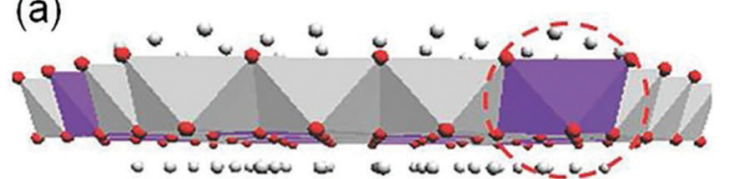

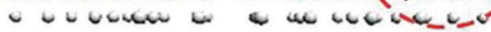
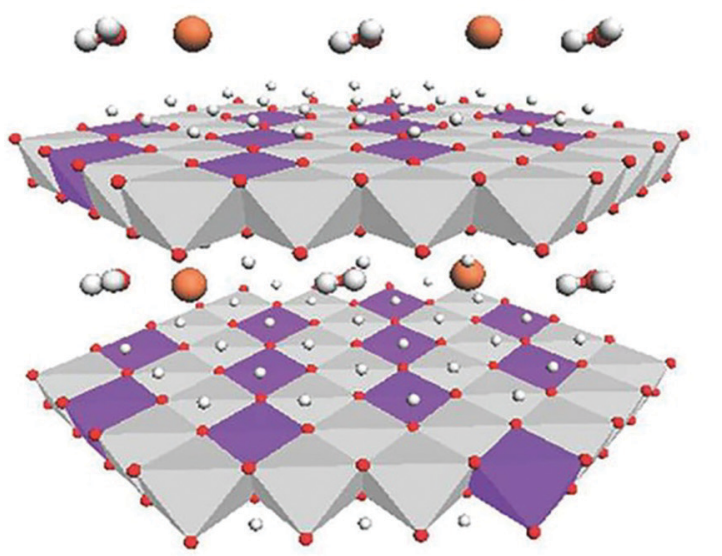

$\mathrm{M}^{3+} \bigcirc \mathrm{M}^{2+} \bigcirc \mathrm{OCH}$ O $\mathrm{H}_{2} \mathrm{O} O$ anion (b)

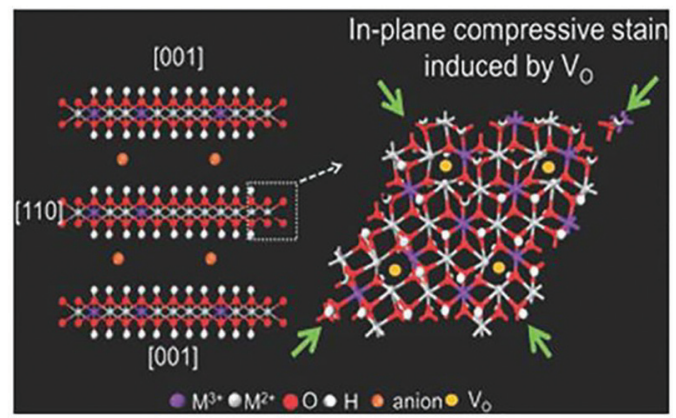

(c)

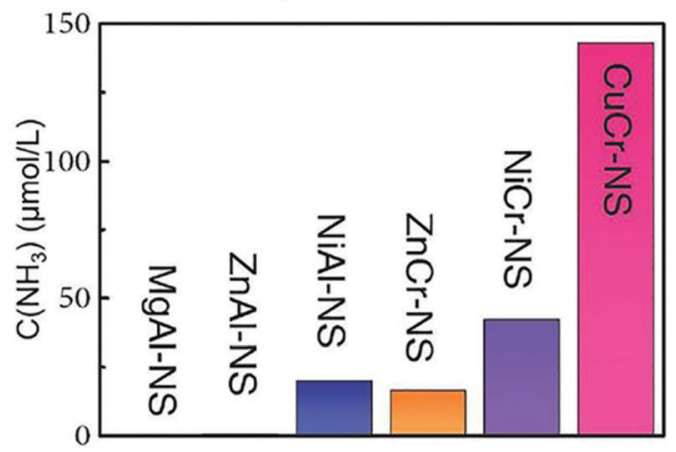

Fig. 4 (a) Schematic representation of the layered LDH structure with defective $\mathrm{MO}_{6}$ octahedra. (b) Schematic of the in-plane biaxial compressive strain in $\mathrm{CuCr}-\mathrm{NS}$. (c) The yield of $\mathrm{NH}_{3}$ over different $\mathrm{LDH}$ photocatalysts under visible-light illumination $(\lambda>400 \mathrm{~nm})$. Reproduced from ref. 34 with permission from Wiley- $\mathrm{VCH}$.

Schrauzer et al. studied the effect of different metal doped $\mathrm{TiO}_{2}$ on the photocatalytic activity of $\mathrm{N}_{2}$ reduction, including iron (Fe), chromium (Cr), cobalt (Co), and molybdenum (Mo). ${ }^{17}$ They found that Fe doping was the most effective for enhancing the photocatalytic activity of $\mathrm{TiO}_{2}$ for photosynthesis of $\mathrm{NH}_{3}$ from $\mathrm{N}_{2}$ and $\mathrm{H}_{2} \mathrm{O}$. However, $\mathrm{O}_{2}$ production as the oxidation product was not reported. In this regard, Zhao et al. investigated the Fe-doped $\mathrm{TiO}_{2}$ with highly exposed (101) facets for $\mathrm{N}_{2}$ photofixation, ${ }^{64}$ and found that an optimum $\mathrm{Fe}^{3+}$ doping content played a key role in inhibiting the recombination of photoinduced electron-hole pairs, which could act as a temporary electron/hole trapping sites, therefore enhancing the concentration of charge carriers and improving the photocatalytic performance. With this in mind, Liu et al. also reported a Fe-doped $\mathrm{SrMoO}_{4}$ (FSMO) as a potential candidate for $\mathrm{N}_{2}$ photoreduction. ${ }^{65}$ Further studies revealed that the intrinsic bandgap of $\mathrm{SrMoO}_{4}$ could be shrunk from $3.98 \mathrm{eV}$ to $2.93 \mathrm{eV}$ with the increase in Fe doping concentration (from 0 to 5.1\%), resulting in the extension of light adsorption from the ultraviolet to the visible-light region. Besides that, the Fe doping could induce the formation of surface defects as active sites for $\mathrm{N}_{2}$ adsorption and significantly retard the recombination of electrons and holes, leading to enhanced $\mathrm{N}_{2}$ reduction reaction. As a result of these properties, an improved $\mathrm{NH}_{3}$ production rate of $93.1 \mu \mathrm{mol} \mathrm{g}{ }^{-1} \mathrm{~h}^{-1}$ over the optimal FSMO was achieved compared with that of pristine $\mathrm{SrMoO}_{4}\left(66.7 \mu \mathrm{mol} \mathrm{g}{ }^{-1} \mathrm{~h}^{-1}\right)$. In another related work incorporating $\mathrm{Fe}$ into $\mathrm{BiOCl}$ nanosheets, Fe-doped BiOCl nanosheets (BiOCl NSs-Fe) were developed for $\mathrm{N}_{2}$ photoreduction. ${ }^{66}$ The optimal BiOCl NSs-Fe exhibited a marked enhancement of photocatalytic $\mathrm{NH}_{3}$ production, and the efficiency was 2.53 times higher than that of pristine BiOCl NSs.

In another study, Mo was successfully doped into $\mathrm{W}_{18} \mathrm{O}_{49}$ nanowires to produce Mo-doped $\mathrm{W}_{18} \mathrm{O}_{49}$ nanowires (MWO-1) as shown in Fig. 7a. ${ }^{67}$ Compared with OV-rich $\mathrm{W}_{18} \mathrm{O}_{49}$ nanowires, Mo-doping had many kinds of effects on photocatalytic $\mathrm{N}_{2}$ reduction. Fig. $7 \mathrm{~b}$ displays the electronic band structures of MWO-1 and $\mathrm{W}_{18} \mathrm{O}_{49}$; it is observed that the defect-band center could be shifted to the Fermi level by Mo doping, which provided more energetic electrons for NRR. Theoretical simulations revealed that the Mo-W centers could alter electron distribution, leading to a larger adsorption energy $(-2.48 \mathrm{eV})$ relative to $\mathrm{W}_{18} \mathrm{O}_{49}$ with $\mathrm{W}-\mathrm{W}$ sites $(1.65 \mathrm{eV})$, which was in favor of $\mathrm{N}_{2}$ chemisorption and activation (Fig. 7c). Additionally, the enhanced $\mathrm{M}-\mathrm{O}$ co-valence caused by Mo doping could effectively promote the electron transfer from metal active centers to adsorbed $\mathrm{N}_{2}$ molecules. Thus, the optimal MWO-1 exhibited an excellent $\mathrm{NH}_{3}$ yield rate of $195.5 \mu \mathrm{mol} \mathrm{g} \mathrm{g}^{-1} \mathrm{~h}^{-1}$ under fullspectrum irradiation (Fig. 7d), which was 7 times higher than that of $\mathrm{W}_{18} \mathrm{O}_{49}$, and also realized a high AQE of $0.33 \%$ at $400 \mathrm{~nm}$.

Furthermore, Cu-doped ultrathin $\mathrm{TiO}_{2}$ nanosheets $\left(\mathrm{TiO}_{2}-\mathrm{V}_{\mathrm{O}}-\right.$ strain) were also studied. ${ }^{68}$ Compared with pristine $\mathrm{TiO}_{2}$, introducing $\mathrm{Cu}$ into $\mathrm{TiO}_{2}$ could create abundant OVs and cause lattice distortion and strain effects, resulting in a significant increase of $\mathrm{N}_{2}$ adsorption energy on the surface of $\mathrm{TiO}_{2}-\mathrm{V}_{\mathrm{O}^{-}}$ strain $(-0.37 \mathrm{eV})$ compared with that of pristine $\mathrm{TiO}_{2}(-0.17 \mathrm{eV}$, Fig. 8a). The increased adsorption energy promoted the electron transfer from $\mathrm{TiO}_{2}$ to $\mathrm{N}_{2}$, thereby breaking the $\mathrm{N} \equiv \mathrm{N}$ bonds. Meanwhile, the required reaction energy for $\mathrm{N}_{2}$ hydrogenation to 

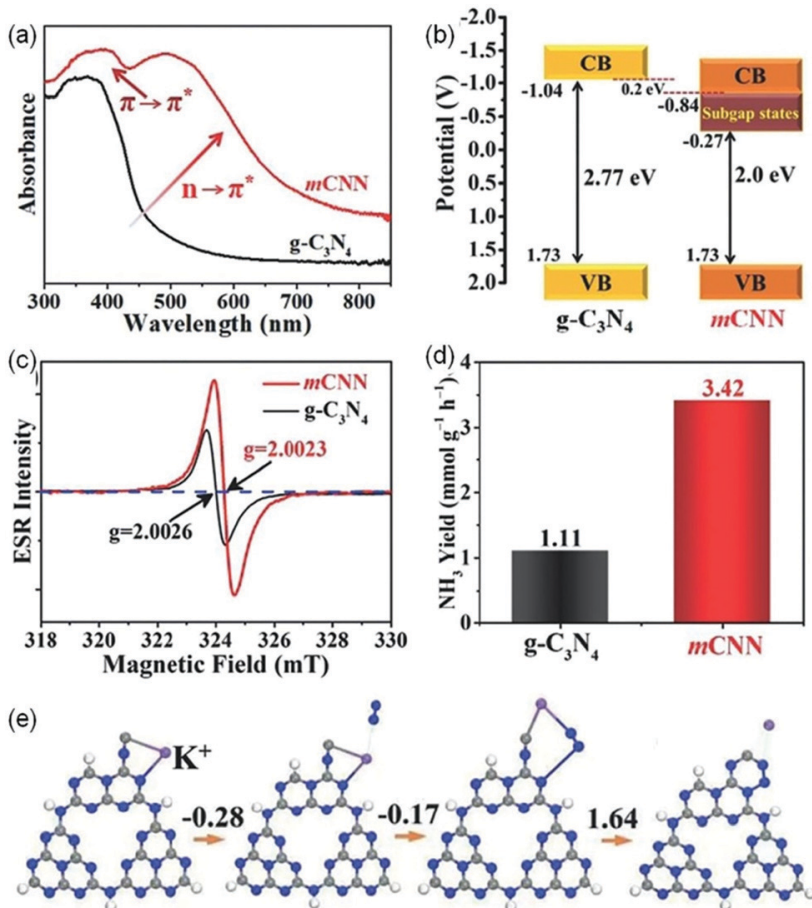

Fig. 5 (a) UV-vis diffuse reflectance spectra (DRS). (b) Schematic illustration of energy band structures. (c) Room-temperature electron paramagnetic resonance (EPR) spectra. (d) $\mathrm{NH}_{3}$ production rates of $\mathrm{g}-\mathrm{C}_{3} \mathrm{~N}_{4}$ and mCNN. (e) The calculated free-energy changes for the NRR pathway using $m C N N$. The blue sphere represents $N$, the grey sphere represents $C$, the white sphere represents $\mathrm{H}$, and the purple sphere represents $\mathrm{K}^{+}$. Reproduced from ref. 55 with permission from Wiley- $\mathrm{VCH}$.

$\mathrm{N}-\mathrm{NH}^{*}$ was only $0.365 \mathrm{eV}$ on $\mathrm{TiO}_{2}-\mathrm{V}_{\mathrm{o}}$-strain, which was lower than that on pristine $\mathrm{TiO}_{2}(2.115 \mathrm{eV}$, Fig. 8b). As a result, the optimal $\mathrm{TiO}_{2}-\mathrm{V}_{\mathrm{o}}$-strain $\left(6 \% \mathrm{TiO}_{2}\right)$ achieved an enhanced $\mathrm{NH}_{3}$ yield rate of $78.9 \mu \mathrm{mol} \mathrm{g}{ }^{-1} \mathrm{~h}^{-1}$ under full solar irradiation, about 5.2 times higher than that of the $\mathrm{TiO}_{2}$ nanosheets with OVs $\left(0 \% \mathrm{TiO}_{2}\right.$, Fig. 8c). In addition, the yield rate of $\mathrm{O}_{2}\left(59.1 \mu \mathrm{mol} \mathrm{g}{ }^{-1} \mathrm{~h}^{-1}\right)$ and $\mathrm{NH}_{3}$ mentioned above over $\mathrm{TiO}_{2}-\mathrm{V}_{\mathrm{o}}$-strain was close to the stoichiometric ratio of $3: 4$, revealing that the protons in $\mathrm{NH}_{3}$ were from the $\mathrm{H}_{2} \mathrm{O}$ molecules. Most recently, a novel bimetallic system with $\mathrm{Fe}-\mathrm{Pt}$ loaded $\mathrm{g}-\mathrm{C}_{3} \mathrm{~N}_{4}$ was used for efficient ammonia synthesis under mild conditions. ${ }^{72}$ Further investigations proposed that the doping of Pt onto the Fe nanocluster over the surface of $\mathrm{g}-\mathrm{C}_{3} \mathrm{~N}_{4}$ could cause an uplift of the energy band of semiconductors and form a large Schottky barrier, thus leading to an improved separation of photogenerated carriers and enhanced $\mathrm{N}_{2}$ reduction. Accordingly, $0.3 \mathrm{wt} \% \mathrm{Pt}$ doped on $3 \mathrm{wt} \%$ Fe@ $\mathrm{C}_{3} \mathrm{~N}_{4}$ exhibited a high $\mathrm{NH}_{3}$ production rate of $63 \mu \mathrm{g} \mathrm{h} \mathrm{h}^{-1} \mathrm{~g}^{-1}$ with gaseous $\mathrm{H}_{2}$ and $\mathrm{N}_{2}$ as reactants under visible light irradiation and the AQE was tested to be $0.15 \%$ between 450 and $500 \mathrm{~nm}$.

Apart from metal doping, a metal-free B-doped g- $\mathrm{C}_{3} \mathrm{~N}_{4}$ nanosheet (BCN) with exposed active $\mathrm{N}$ atoms was recently synthesized for highly efficient ammonia synthesis. ${ }^{73}$ Theoretical studies revealed that the exposed $\mathrm{N}$ atoms could be stabilized by $\mathrm{B}-\mathrm{N}-\mathrm{C}$ coordination on $\mathrm{BCN}$, which was different from that in the pristine $\mathrm{g}-\mathrm{C}_{3} \mathrm{~N}_{4}$. The $\mathrm{B}$ dopants were proved as active sites for $\mathrm{N}_{2}$ adsorption and activation and could effectively retard charge recombination and improve light utilization. The optimal BCN with $13.8 \mathrm{wt} \%$ B-dopants exhibited a remarkable $\mathrm{NH}_{3}$ yield rate of $313.9 \mu \mathrm{mol} \mathrm{g}{ }^{-1} \mathrm{~h}^{-1}$ under visible light irradiation in the presence of $\mathrm{Na}_{2} \mathrm{SO}_{3}$ as a hole scavenger, which was much higher than that $\left(32.8 \mu \mathrm{mol} \mathrm{g}{ }^{-1} \mathrm{~h}^{-1}\right)$ over pristine $\mathrm{g}-\mathrm{C}_{3} \mathrm{~N}_{4}$ and also achieved a good QE of around $0.64 \%$ at $420 \mathrm{~nm}$. In addition, carbon-doped $\mathrm{TiO}_{2}$ was also reported for $\mathrm{N}_{2}$ photoreduction, which exhibited an $\mathrm{NH}_{3}$ yield rate of $109.3 \mu \mathrm{mol} \mathrm{g}{ }^{-1} \mathrm{~h}^{-1}$.

\section{Morphology engineering}

Facets. Since the surface atomic distribution has a huge effect on the active sites and electronic structures of photocatalysts, ${ }^{69,74-76}$ many researchers attempted to control the crystal facet of semiconductors to enhance the photocatalytic $\mathrm{N}_{2}$ reduction. For instance, two $\mathrm{Bi}_{5} \mathrm{O}_{7} \mathrm{I}$ nanosheets with different $\{100\}$ and $\{001\}$ facets were synthesized successfully via hydrolysis and calcination methods, respectively. ${ }^{77}$ Further investigations
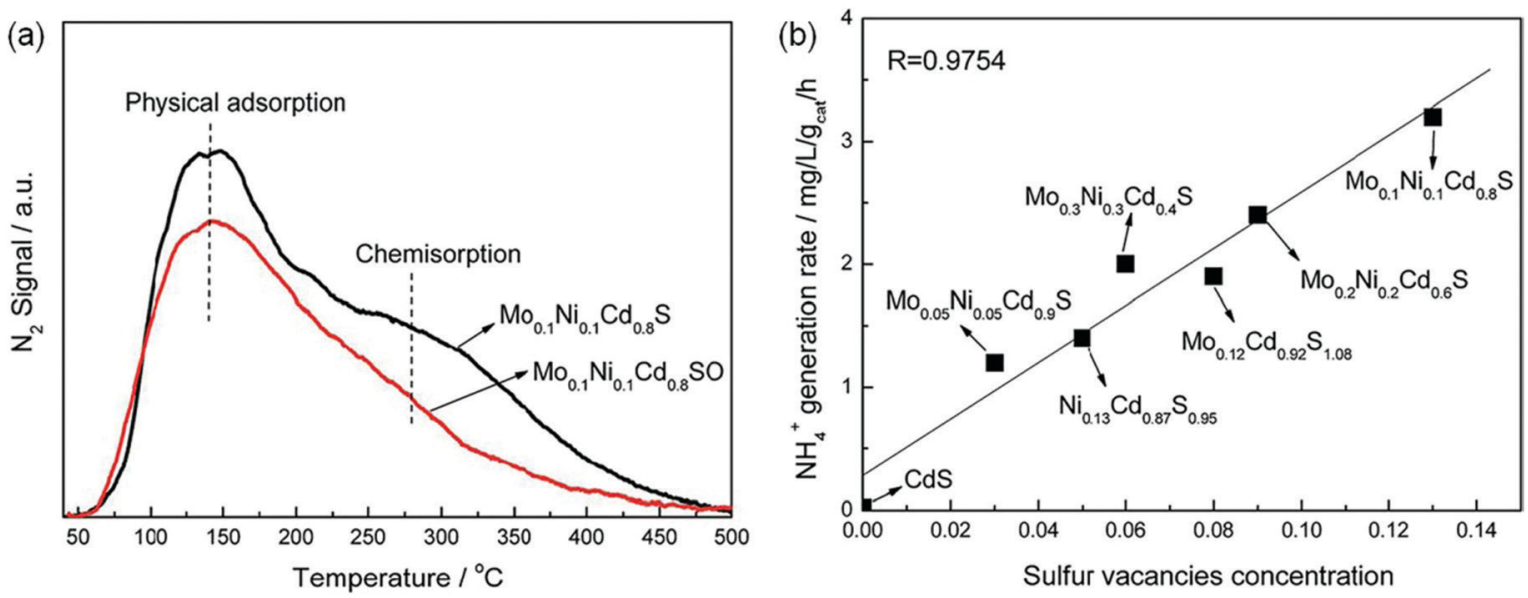

Fig. 6 (a) The $\mathrm{N}_{2}$-TPD of $\mathrm{Mo}_{0.1} \mathrm{Ni}_{0.1} \mathrm{Cd}_{0.8} \mathrm{~S}$ and $\mathrm{Mo}_{0.1} \mathrm{Ni}_{0.1} \mathrm{Cd}_{0.8} \mathrm{~S}$. (b) The relationship of $\mathrm{NH}_{3}$ yield over the obtained photocatalysts and the SV concentration. Reproduced from ref. 57 with permission from The Royal Society of Chemistry. 
(a)

(c)
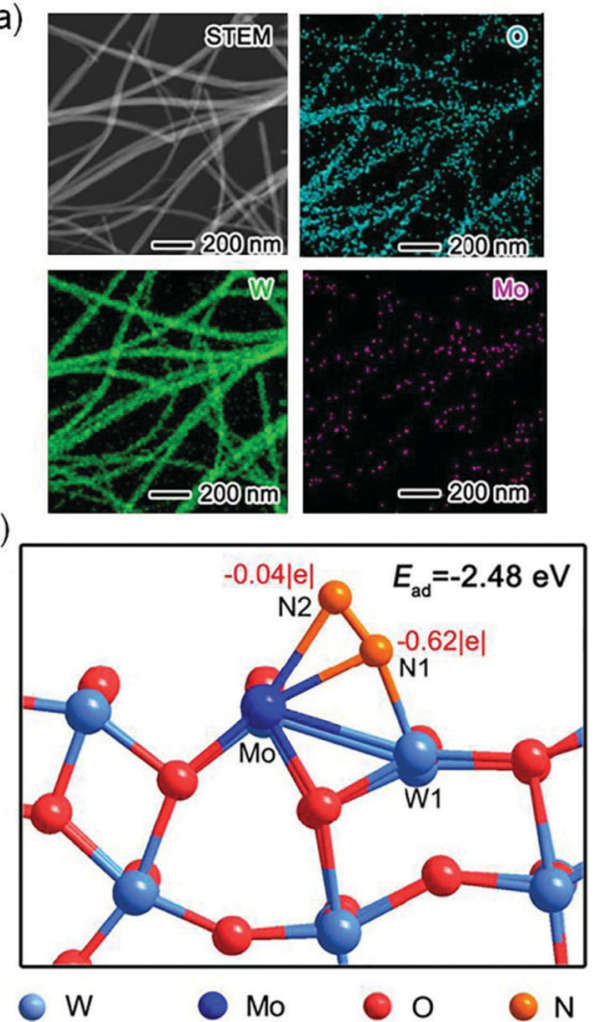

(b)

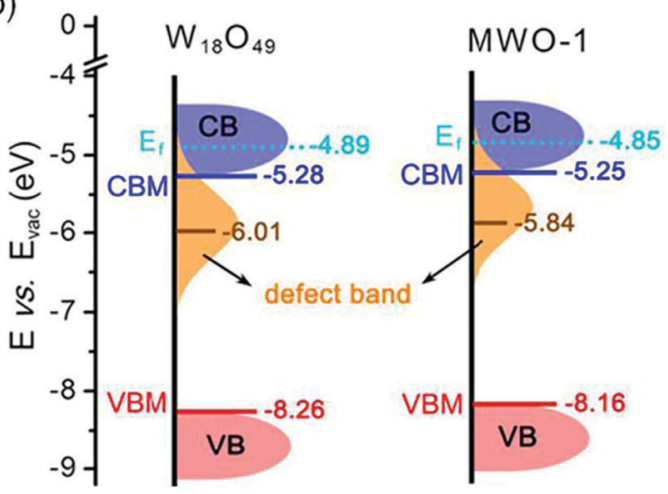

(d)

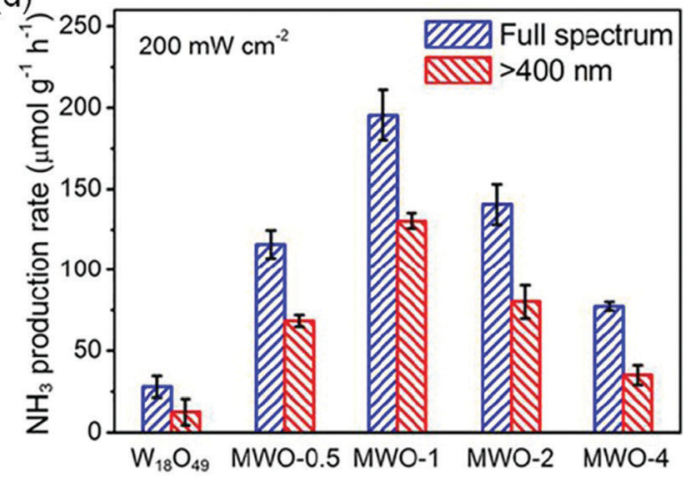

Fig. 7 (a) The scanning TEM (STEM) image and the corresponding elemental mapping of MWO-1. (b) Schematic illustration of the band structures of $\mathrm{W}_{18} \mathrm{O}_{49}$ and MWO-1. (c) Schematic simulation for $\mathrm{N}_{2}$ adsorption and activation on the surface active sites of the Mo-doped $\mathrm{W}_{18} \mathrm{O}_{49}$ model. (d) The photocatalytic $\mathrm{NH}_{3}$ yield on various catalysts. Reproduced from ref. 67 with permission from ACS Publication.

revealed that $\mathrm{Bi}_{5} \mathrm{O}_{7} \mathrm{I}-001$ had more negative conduction band position $(-1.45 \mathrm{eV})$ compared with that in $\mathrm{Bi}_{5} \mathrm{O}_{7} \mathrm{I}-100$ $(-0.85 \mathrm{eV})$ and exhibited higher efficiency for separation of photoinduced carriers, thus leading to a high photocatalytic activity for $\mathrm{N}_{2}$ photoreduction. Accordingly, a remarkable $\mathrm{NH}_{3}$ generation rate of $111.5 \mu \mathrm{mol} \mathrm{g}{ }^{-1} \mathrm{~h}^{-1}$ over $\mathrm{Bi}_{5} \mathrm{O}_{7} \mathrm{I}-001$ was achieved using methanol as a hole scavenger and the $\mathrm{AQE}$ of that was increased to $5.1 \%$.

In another report, Zhang et al. also systematically investigated the influence of $\{001\}$ and $\{010\}$ facets of OV-rich BiOCl nanosheets on $\mathrm{N}_{2}$ adsorption and activation. ${ }^{78}$ Experimental results showed that $\{010\}$ facet BiOCl nanosheets (BOC-010) exhibited a superior performance with a $\mathrm{NH}_{3}$ yield of $4.62 \mu \mathrm{mol} \mathrm{g}^{-1} \mathrm{~h}^{-1}$, which was around 2.5 times higher than that on $\{001\}$ facet BiOCl nanosheets (BOC-001, Fig. 9a). Meanwhile, it was observed that $\mathrm{N}_{2} \mathrm{H}_{4}$ which served as a main intermediate was accumulated on BOC-010 during $\mathrm{N}_{2}$ photoreduction reaction within $30 \mathrm{~min}$ and was consumed gradually to be converted into $\mathrm{NH}_{3}$ (Fig. 9b). Importantly, when $\mathrm{N}_{2} \mathrm{H}_{4}$ was used as the reactant, both BOC-010 and BOC-001 showed a similar photocatalytic conversion efficiency of $\mathrm{N}_{2} \mathrm{H}_{4}$ under simulated solar irradiation (Fig. 9c), which in turn meant that BOC-010 had a stronger ability to generate $\mathrm{N}_{2} \mathrm{H}_{4}$ intermediates. Further DFT calculations revealed that the $\mathrm{N}_{2}$ fixation on BOC-010 followed an alternative pathway with $\mathrm{N}_{2} \mathrm{H}_{4}$ as the main intermediate, which could provide a lower energy pathway for $\mathrm{N}_{2}$ fixation via proton-coupled electron transfer compared with that via a distal pathway occurring on BOC-001 facets, thus resulting in enhanced $\mathrm{NH}_{3}$ production (Fig. 9d and e).

Nanostructure engineering. Nanomaterials have preternatural interface structures and unique functions, such as the small size effect, the surface and boundary effect, quantum size and so on. ${ }^{79-85}$ In order to increase the catalytic active sites of the photocatalytic reduction of $\mathrm{N}_{2}$, Sun et al. synthesized BiO quantum dots with an average size of 2-5 nm (Fig. 10a), which showed an $\mathrm{NH}_{3}$ production rate of $1226 \mu \mathrm{mol} \mathrm{g}^{-1} \mathrm{~h}^{-1}$ without a cocatalyst and a sacrificial agent. ${ }^{86}$ Meanwhile, the amount of produced $\mathrm{O}_{2}$ increased continuously with increasing irradiation time, consistent with the stoichiometric chemistry. Kinetic analysis and quantum chemical calculations suggested that the highly efficient photocatalytic activity of BiO quantum dots could be attributed to the fact that the smaller size endowed the surface and edge of $\mathrm{BiO}$ rich in $\mathrm{Bi}^{2+}$ species, which had the potential to increase the electron donation to the anti-bonding $\pi^{*}$ orbitals of $\mathrm{N}_{2}$, thereby could act as active sites to adsorb and activate $\mathrm{N}_{2}$ (Fig. 10b). More recently, a tubular OV-rich $\mathrm{Bi}_{5} \mathrm{O}_{7} \mathrm{Br}$ $\left(\mathrm{Bi}_{5} \mathrm{O}_{7} \mathrm{Br}-\mathrm{NT}\right)$ with a diameter of $5 \mathrm{~nm}$ (Fig. 10c) was prepared and it exhibited a maximum $\mathrm{NH}_{3}$ yield rate of $1.38 \mathrm{mmol} \mathrm{g}^{-1} \mathrm{~h}^{-1}$ (Fig. 10d) upon changing the OV concentration. ${ }^{87} \mathrm{~A}$ high specific surface area of up to $96.56 \mathrm{~m}^{2} \mathrm{~g}^{-1}$ was observed for $\mathrm{Bi}_{5} \mathrm{O}_{7} \mathrm{Br}-\mathrm{NT}$, which indicated the exposure of abundant surface 


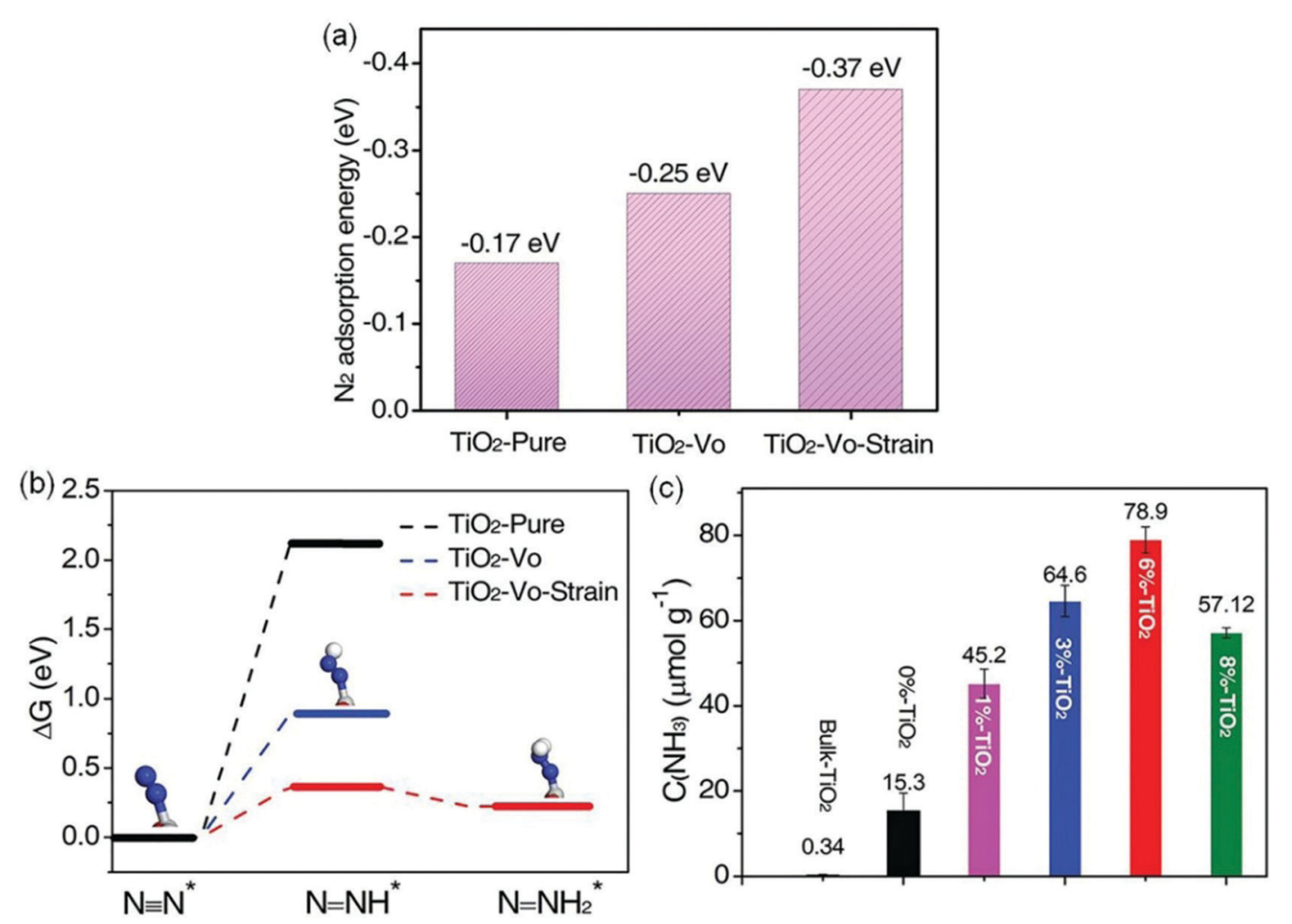

Fig. 8 (a) The $\mathrm{N}_{2}$ adsorption energies by DFT calculations on different photocatalysts. (b) The calculated Gibbs free energy for $\mathrm{N}_{2}$ reduction on the (001) surface of different photocatalysts ( $\mathrm{H}$ : white sphere, Ti: gray sphere, $\mathrm{N}$ : blue sphere, O: red sphere). (c) Yield of $\mathrm{NH}_{3}$ Over various samples under UV-vis irradiation. Reproduced from ref. 68 with permission from Wiley-VCH.

OVs for the chemisorption and activation of $\mathrm{N}_{2}$. It was found that the OVs on the $\mathrm{Bi}_{5} \mathrm{O}_{7} \mathrm{Br}$-NT surface could not only effectively promote $\mathrm{N}_{2}$ adsorption and activation, but also could be regenerated by capturing $\mathrm{O}$ atoms from $\mathrm{H}_{2} \mathrm{O}$ after the reaction to maintain the good stability of $\mathrm{Bi}_{5} \mathrm{O}_{7} \mathrm{Br}$ nanostructures. As a result, the calculated $\mathrm{AQE}$ for the $\mathrm{Bi}_{5} \mathrm{O}_{7} \mathrm{Br}-\mathrm{NT}$ photocatalyst was $2.3 \%$ under visible light irradiation at $420 \mathrm{~nm}$ (Fig. 10e). Furthermore, it was reported that metal-free black phosphorus nanoflakes with abundant edges (eBP NFs) synthesized via a facile chemical etching exfoliation method exhibited a remarkable $\mathrm{NH}_{3}$ yield rate of $2.37 \mathrm{mmol} \mathrm{g}{ }^{-1} \mathrm{~h}^{-1}$ under visible light irradiation in the presence of $\mathrm{Na}_{2} \mathrm{SO}_{3}$ and $\mathrm{Na}_{2} \mathrm{~S} \cdot 9 \mathrm{H}_{2} \mathrm{O}$ as scavengers. ${ }^{88}$ The photoelectrochemical characteristics (PEC) and transient absorption (TA) studies revealed that the efficient ammonia synthesis was attributed to rich edges on the surface, which could provide abundant active sites for enhanced $\mathrm{N}_{2}$ adsorption and activation. Similarly, a nanocomposite $\left(\mathrm{SiO}_{2} / \mathrm{C}-\mathrm{RP}\right)$ prepared by loading red phosphorus on $\mathrm{SiO}_{2}$ nanospheres showed superior charge separation. ${ }^{89}$ Besides, this hybrid nanostructure exhibited a large surface area, good water dispersibility and large light adsorption. As a result of these benefits, $\mathrm{SiO}_{2} / \mathrm{C}-\mathrm{RP}$ exhibited an $\mathrm{NH}_{3}$ production yield of $0.73 \mu \mathrm{mol} \mathrm{h}^{-1}$ under full-spectrum irradiation.

\section{Interfacial modulation}

Cocatalyst loading. With the similar idea of using metal cocatalysts to inhibit the recombination of electrons and holes and enhance the photostability of catalysts in other photocatalytic processes, e.g. water splitting, ${ }^{90-102}$ Ranjit et al. studied different metal loaded $\mathrm{TiO}_{2}$ toward the photoreduction of $\mathrm{N}_{2}$, including ruthenium $(\mathrm{Ru})$, rhodium $(\mathrm{Rh})$, palladium $(\mathrm{Pd})$, and platinum $(\mathrm{Pt}) \cdot{ }^{93}$ It was found that the order of the photocatalytic activity of these metals on $\mathrm{TiO}_{2}$ was $\mathrm{Ru}>\mathrm{Rh}>\mathrm{Pd}>$ Pt. The excellent performance of Ru-loaded $\mathrm{TiO}_{2}$ could be assigned to the high metal-hydrogen bond strength which well correlated with the high production of $\mathrm{NH}_{3}$. In recent years, singleatom catalysts (SACs) have been well studied as excellent candidates for boosting the catalytic activity and improving the utilization efficiency of metal atoms during the photocatalytic process. ${ }^{94}$ To further improve the performance of the photocatalytic $\mathrm{NH}_{3}$ synthesis, a single atomic Ru decorated $\mathrm{TiO}_{2}$ nanosheet with abundant OVs was prepared..$^{95}$ The DFT calculations indicated that the single atomic $\mathrm{Ru}$ species were favorable to be formed and dispersed on the OVs over the $\mathrm{TiO}_{2}$ nanosheet surface. Further investigations revealed that the isolated $\mathrm{Ru}$ atom could serve as the active site for $\mathrm{N}_{2}$ adsorption and activation, promote the separation of photogenerated charge carriers efficiently, as well as inhibit the competitive $\mathrm{H}_{2}$ evolution reaction, thus leading to an improved $\mathrm{NH}_{3}$ photosynthesis. Thus, a $1 \mathrm{wt} \%$ single $\mathrm{Ru}$ atom decorated $\mathrm{TiO}_{2}$ nanosheet exhibited a superior performance with an $\mathrm{NH}_{3}$ yield of $56.3 \mu \mathrm{g} \mathrm{h}^{-1} \mathrm{~g}_{\text {cat }}{ }^{-1}$, which was two times as high as that of pure $\mathrm{TiO}_{2}$. Recently, Au-anchored $\mathrm{TiO}_{2}$ nanosheets with $\mathrm{OV}\left(\mathrm{Au} / \mathrm{TiO}_{2}-\mathrm{OV}\right)$ photocatalysts were developed for $\mathrm{N}_{2}$ photofixation. ${ }^{96}$ As shown in Fig. 11a, Au nanospheres 

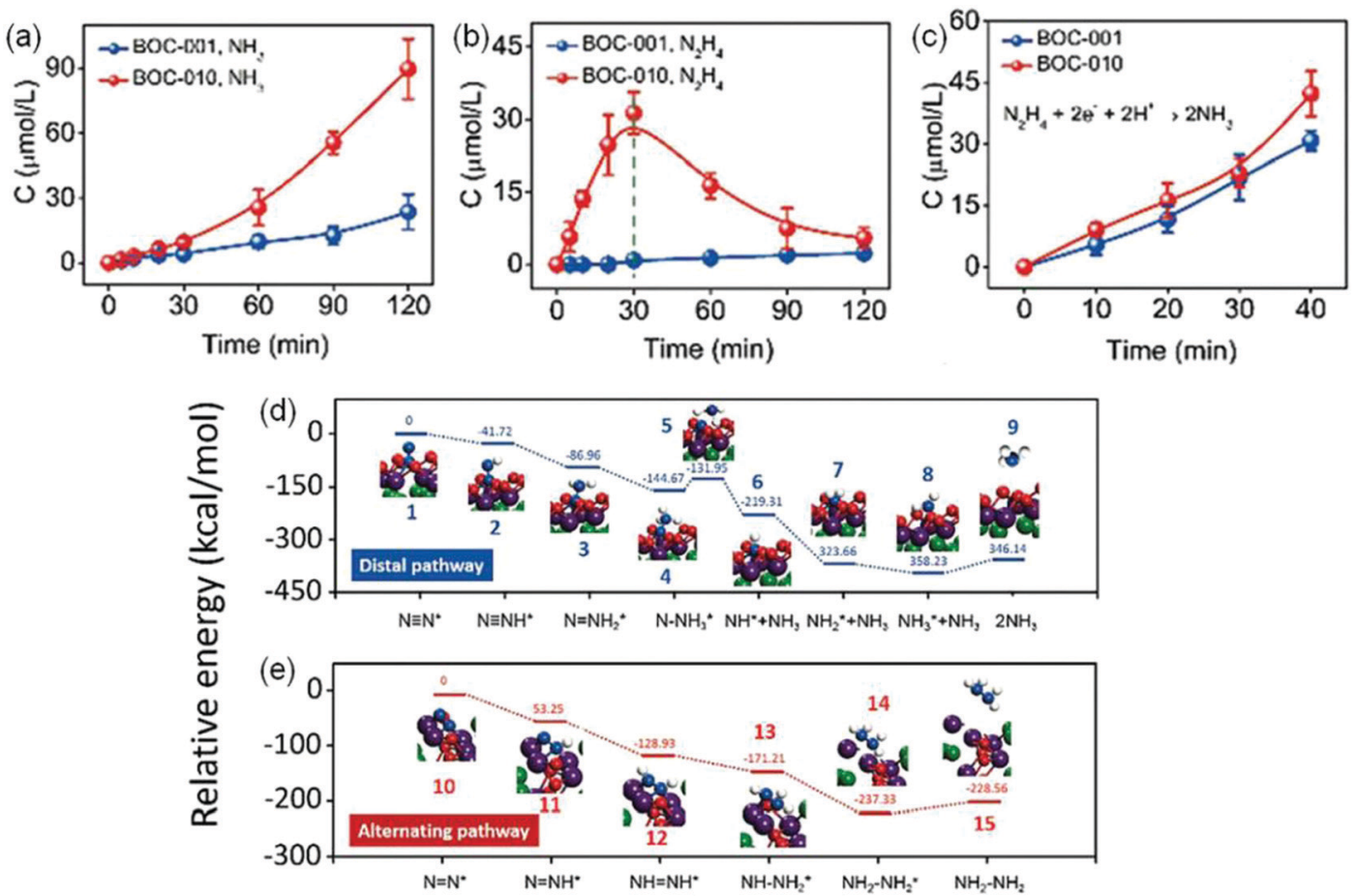

Fig. 9 Photocatalytic performance for $\mathrm{N}_{2}$ reduction on BOC-001 and BOC-010. The yield comparison of produced (a) $\mathrm{NH}_{3}$ and (b) $\mathrm{N}_{2} \mathrm{H}_{4}$ on $\mathrm{BOC}-001$ and BOC-010 under simulated solar light irradiation. (c) The $\mathrm{NH}_{3}$ yield produced from $\mathrm{N}_{2} \mathrm{H}_{4} \mathrm{BOC}-001$ and $\mathrm{BOC}-010$ under simulated solar light irradiation. The calculated free energy change for $\mathrm{N}_{2}$ reduction (d) on the (001) surface via a distal pathway and (e) on the (010) surface via an alternating pathway. Reproduced from ref. 78 with permission from The Royal Society of Chemistry.

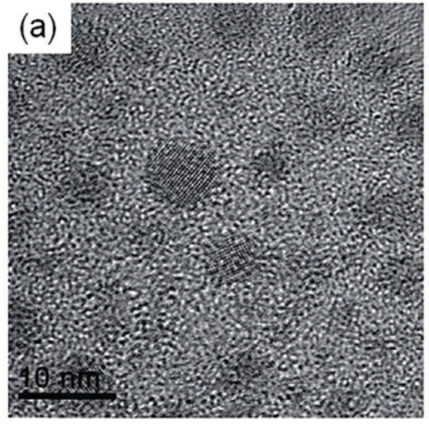

(b)
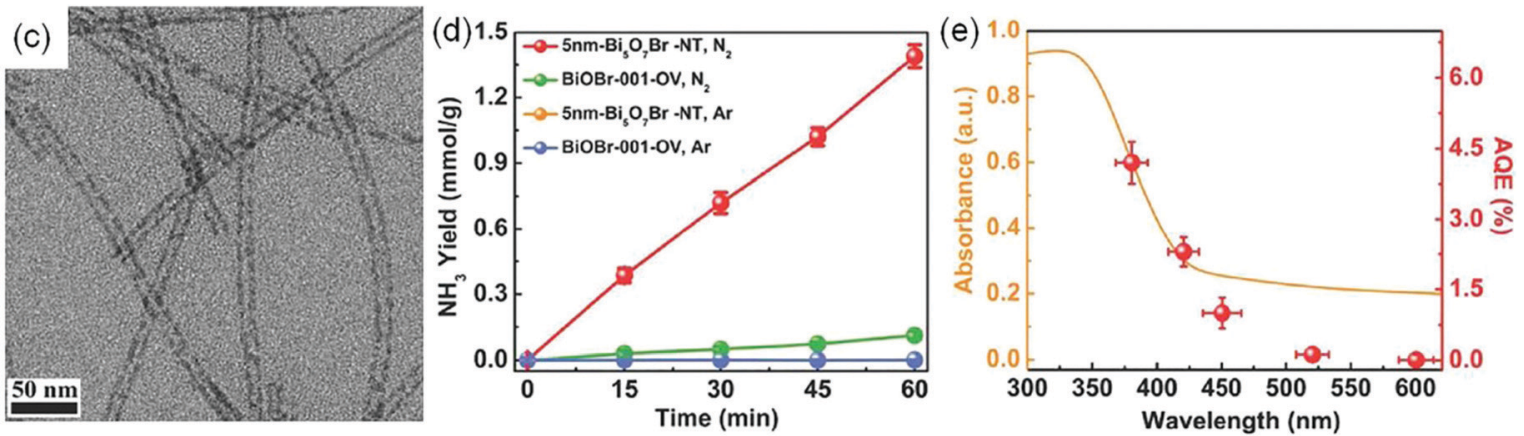

Fig. 10 (a) TEM image of $\mathrm{BiO}$ quantum dots. (b) Proposed mechanism for $\mathrm{N}_{2}$ photoreduction on $\mathrm{BiO}$ quantum dots. Reproduced from ref. 86 with permission from The Royal Society of Chemistry. (c) TEM image of $\mathrm{Bi}_{5} \mathrm{O}_{7} \mathrm{Br}-\mathrm{NT}$. (d) Yield of $\mathrm{NH}_{3}$ for $\mathrm{Bi}_{5} \mathrm{O}_{7} \mathrm{Br}-\mathrm{NT}$. (e) The wavelength-dependent AQE of $\mathrm{Bi}_{5} \mathrm{O}_{7} \mathrm{Br}-\mathrm{NT}$. Reproduced from ref. 87 with permission from Wiley-VCH.

with a diameter of $20 \mathrm{~nm} \pm 1.4 \mathrm{~nm}$ were uniformly distributed on the surface of $\mathrm{TiO}_{2}$-OV nanosheets. The UV-vis spectrum of
$\mathrm{Au} / \mathrm{TiO}_{2}$-OV showed absorption characteristics of $\mathrm{Au}$ loaded $\mathrm{TiO}_{2}\left(\mathrm{Au} / \mathrm{TiO}_{2}\right)$ and $\mathrm{TiO}_{2}$-OV, displaying a broad localized 
surface plasmon resonance (LSPR) peak at $550 \mathrm{~nm}$ and an obvious absorption tail (Fig. 11b). Therefore, in the $\mathrm{Au} / \mathrm{TiO}_{2}-\mathrm{OV}$ hybrid, OVs on the surface of $\mathrm{TiO}_{2}$ nanosheets chemisorbed and activated $\mathrm{N}_{2}$ molecules, which were reduced to $\mathrm{NH}_{3}$ by plasmon-induced hot electrons from $\mathrm{Au}$ nanoparticles in the presence of methanol as a hole scavenger (Fig. 11c). As a result, the optimal $\mathrm{Au} / \mathrm{TiO}_{2}$-OV exhibited an $\mathrm{NH}_{3}$ photoproduction rate of $78.6 \mu \mathrm{mol} \mathrm{g}{ }^{-1} \mathrm{~h}^{-1}$, which was 98 and 35 times higher than that of $\mathrm{Au} / \mathrm{TiO}_{2}$ and $\mathrm{TiO}_{2}-\mathrm{OV}$ respectively (Fig. 11d). Recently, we reported a hybrid of $2 \mathrm{D}$ layered $\mathrm{Ti}_{3} \mathrm{C}_{2}$ and $\mathrm{P} 25 \mathrm{TiO}_{2}\left(\mathrm{Ti}_{3} \mathrm{C}_{2}-\mathrm{P} 25\right)$, in which $\mathrm{Ti}_{3} \mathrm{C}_{2}$ served as the cocatalyst to facilitate charge separation due to its excellent electrical conductivity. The DFT calculation (Fig. 11e) revealed that the $\mathrm{N}_{2}$ adsorption energies were ranked as $\mathrm{Ti}_{3} \mathrm{C}_{2}(2.731 \mathrm{eV})>\mathrm{VOs}^{-} \mathrm{TiO}_{2}(0.342 \mathrm{eV})>\mathrm{TiO}_{2}$
(0.170 eV), implying that $\mathrm{Ti}_{3} \mathrm{C}_{2}$ had a much stronger $\mathrm{N}_{2}$ chemisorption effect compared with $\mathrm{VOs}_{-} \mathrm{TiO}_{2}$ and $\mathrm{TiO}_{2}$. Therefore the introduction of $\mathrm{Ti}_{3} \mathrm{C}_{2}$ on $\mathrm{P} 25$ could accelerate $\mathrm{N}_{2}$ chemisorption, leading to five times higher photocatalytic yield of $\mathrm{NH}_{3}$ than that on pure P25. More importantly, water acted as the electron donor in this study and stoichiometric $\mathrm{O}_{2}$ production was also observed, which was one of a few examples that could observe the stoichiometric reduction and oxidation products. $^{103}$

In addition to $\mathrm{TiO}_{2}$-based photocatalysts, Pt-loaded $\mathrm{ZnO}$ was reported as an efficient photocatalyst for $\mathrm{N}_{2}$ photoreduction which exhibited an $\mathrm{NH}_{3}$ yield rate of $860 \mathrm{mmol} \mathrm{g}^{-1} \mathrm{~h}^{-1} \cdot{ }^{104}$ More recently, O-doped $1 \mathrm{~T}-\mathrm{MoS}_{2}$ nanosheets with a large amount of SVs $\left(\mathrm{SV}-1 \mathrm{~T}-\mathrm{MoS}_{2}\right)$ were used as cocatalysts over CdS nanorods for (a)

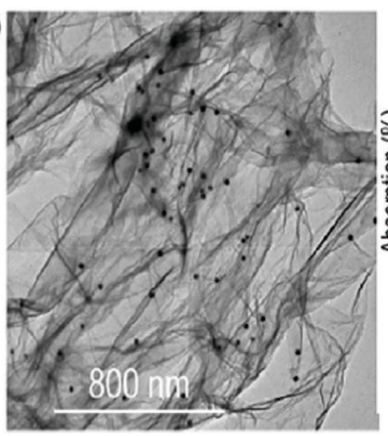

(c)

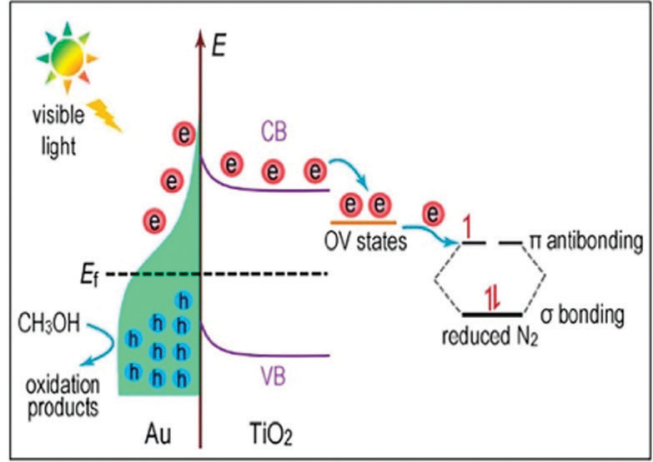

(f)

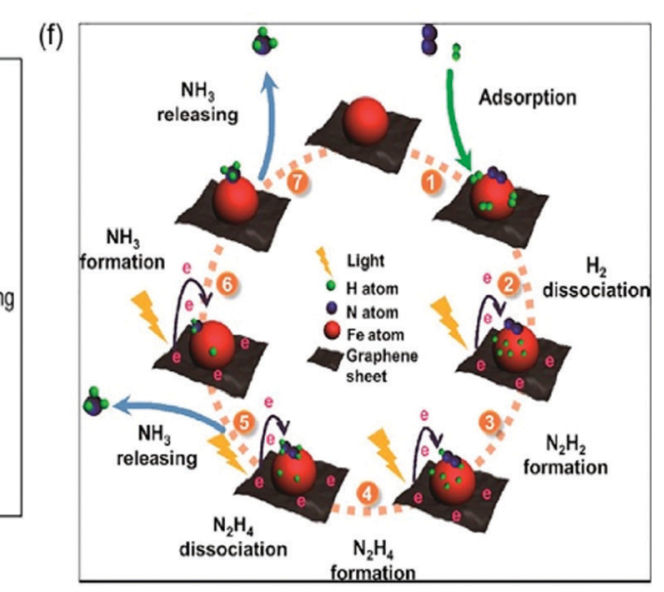

(e)
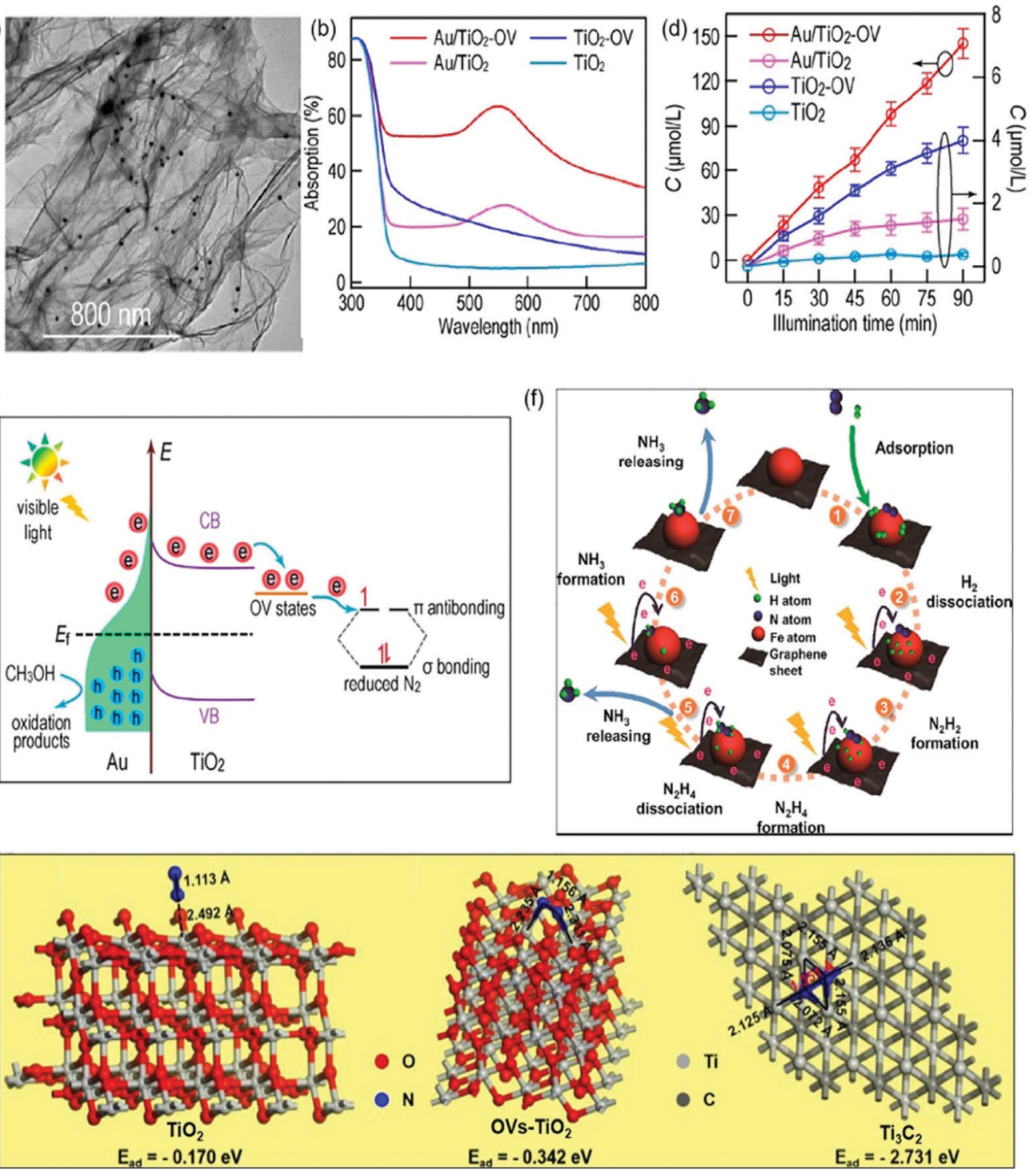

Fig. 11 (a) TEM image of $\mathrm{Au} / \mathrm{TiO}_{2}-\mathrm{OV}$. (b) Adsorption spectra of different photocatalysts. (c) The proposed schematic for photocatalytic $\mathrm{N}_{2}$ reduction over the $\mathrm{Au} / \mathrm{TiO}_{2}-\mathrm{OV}$ catalyst under visible-light irradiation. (d) Photocatalytic $\mathrm{NH}_{3}$ production with different photocatalysts under visible light irradiation. Reproduced from ref. 96 with permission from ACS Publication. (e) Schematic of the adsorption structure on $\mathrm{TiO}_{2}(\mathrm{~A}), \mathrm{OVs}^{-} \mathrm{TiO}_{2}(\mathrm{~B})$ and $\mathrm{Ti}_{3} \mathrm{C}_{2} \mathrm{MXenes}(\mathrm{C})$. Reproduced from ref. 103 with permission from Elsevier Publication. (f) Schematic for photocatalytic $\mathrm{N}_{2}$ reduction over Fe@graphene photocatalysts. Reproduced from ref. 108 with permission from ACS Publication. 
photocatalytic $\mathrm{N}_{2}$ reduction. ${ }^{105}$ The DFT calculations revealed that the SVs and the metal properties of $1 \mathrm{~T}-\mathrm{MoS}_{2}$ could effectively promote the separation of $\mathrm{h}^{+} / \mathrm{e}^{-}$and the presence of SV-1T-MoS ${ }_{2}$ could also provide abundant active sites, resulting in enhanced $\mathrm{N}_{2}$ adsorption and immobilization. Accordingly, a remarkable performance over the optimal SV-1T-MoS ${ }_{2} / \mathrm{CdS}$ was realized with a superior $\mathrm{NH}_{3}$ yield rate of $8220.83 \mu \mathrm{mol} \mathrm{L} \mathrm{L}^{-1} \mathrm{~h}^{-1} \mathrm{~g}^{-1}$ under simulated solar light irradiation. Besides, NiS was also studied as a cocatalyst over CdS nanorods for ammonia photosynthesis. ${ }^{106}$ The DFT calculation indicated that the NiS cocatalyst could reduce the adsorption energy of $\mathrm{N}_{2}$ from $1.16 \mathrm{eV}$ on the CdS surface to $-0.55 \mathrm{eV}$ on the Ni-doped CdS surface. Further characterization revealed that charge separation could also be enhanced by loading NiS on the CdS surface. Because of these properties, a favorable $\mathrm{NH}_{3}$ yield of $2.8 \mathrm{mg} \mathrm{L}^{-1}$ was reported within the first hour in pure water under fullspectrum irradiation. Recently, single-atom Pt modified triazine framework (CTF) nanosheets (Pt-SACs/CTF) were developed for photocatalytic $\mathrm{NH}_{3}$ production. ${ }^{107}$ It was found that the Pt- $\mathrm{N}_{3}$ sites were formed in Pt-SACs/CTF, leading to more negative conduction band position with accelerated thermodynamics and fast interfacial charge migration ability, and therefore a high $\mathrm{NH}_{3}$ production rate of $171.4 \mu \mathrm{mol} \mathrm{g}{ }^{-1} \mathrm{~h}^{-1}$ under visible light irradiation. Most recently, we synthesized a ternary $\mathrm{Ru} / \mathrm{RuO}_{2} / \mathrm{g}-\mathrm{C}_{3} \mathrm{~N}_{4}$ system for $\mathrm{N}_{2}$ photoreduction, which exhibited a photocatalytic $\mathrm{NH}_{3}$ yield of $13.3 \mu \mathrm{mol} \mathrm{g}^{-1} \mathrm{~h}^{-1}$ whilst no $\mathrm{NH}_{3}$ could be measured for pure $\mathrm{g}-\mathrm{C}_{3} \mathrm{~N}_{4}$ under the same condition. Such significant improvement in photocatalytic $\mathrm{NH}_{3}$ synthesis for $\mathrm{Ru} / \mathrm{RuO}_{2} / \mathrm{g}-\mathrm{C}_{3} \mathrm{~N}_{4}$ was not only because of $\mathrm{Ru}$ and $\mathrm{RuO}_{2}$ acting as cocatalysts to promote electron and hole transfer respectively, but also due to the advantage of $\mathrm{Ru}$ for $\mathrm{N}_{2}$ chemisorption and activation. ${ }^{108}$ Besides that, carbon-based materials especially graphene are also often used as cocatalysts to prevent the photoinduced electron-hole recombination and promote the electron transfer due to their prominent electrical conductivity. ${ }^{109,110}$ For instance, a Fe modified three-dimensional graphene (Fe@graphene) photocatalyst was reported for $\mathrm{NH}_{3}$ production. ${ }^{110}$ In the Fe@graphene system (Fig. 11f), graphene generated hot electrons under light irradiation and transferred those electrons to $\mathrm{Fe}$, while $\mathrm{Fe}$ acted as an electron sink and provided the catalytic active sites for the adsorption and activation of $\mathrm{N}_{2}$.

Junction structure. The combination of two semiconductors as a photocatalyst would overcome the shortages of each component, and construct the heterostructure or Z-scheme system with improved photoelectric properties for broad applications. ${ }^{111-115}$ In one work, $\mathrm{Bi}_{2} \mathrm{MoO}_{6}$ was implanted on $\mathrm{g}^{-} \mathrm{C}_{3} \mathrm{~N}_{4}$ nanosheets to enhance the photocatalytic $\mathrm{N}_{2}$ reduction. ${ }^{111}$ In this case, the obtained $\mathrm{g}-\mathrm{C}_{3} \mathrm{~N}_{4} / \mathrm{Bi}_{2} \mathrm{MoO}_{6}$ heterojunction showed effective separation of charge carriers and enhanced photocatalytic performance for $\mathrm{NH}_{3}$ production. In another report, MXene-derived $\mathrm{TiO}_{2} @ \mathrm{C}$ incorporated with $\mathrm{g}-\mathrm{C}_{3} \mathrm{~N}_{4}$ ( $\mathrm{TiO}_{2} @ \mathrm{C} /$ g- $\mathrm{C}_{3} \mathrm{~N}_{4}$, Fig. 12a) was prepared for efficient $\mathrm{NH}_{3}$ photocatalysis. ${ }^{112}$ Compared with the pristine g- $\mathrm{C}_{3} \mathrm{~N}_{4}, \mathrm{TiO}_{2} @ \mathrm{C} / \mathrm{g}-\mathrm{C}_{3} \mathrm{~N}_{4}$ showed a significantly decreased PL intensity (Fig. 12b), which indicated that the heterostructure promoted the charge transfer, which in turn inhibited the recombination of photoinduced electronhole pairs. Furthermore, the transient photocurrent density of $\mathrm{TiO}_{2} @ \mathrm{C} / \mathrm{g}-\mathrm{C}_{3} \mathrm{~N}_{4}$ was about 1.7 times higher than that of $\mathrm{g}-\mathrm{C}_{3} \mathrm{~N}_{4}$ (Fig. 12c), suggesting the improved separation efficiency of the charge carriers. The $\mathrm{N}_{2}$-TDP result revealed that the $\mathrm{Ti}^{3+}$ species on $\mathrm{TiO}_{2}$ and carbonaceous components were responsible (a)

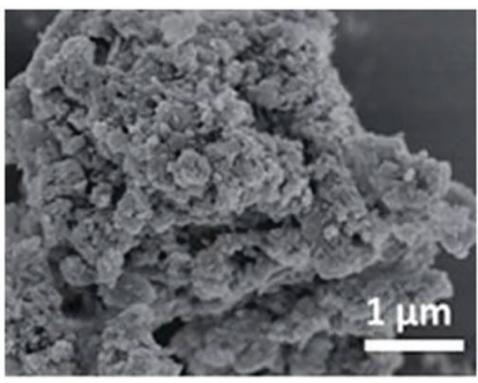

(b)

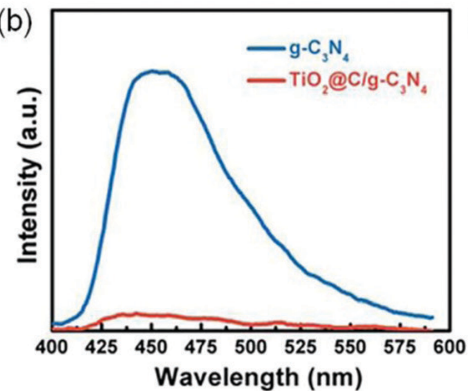

(c)

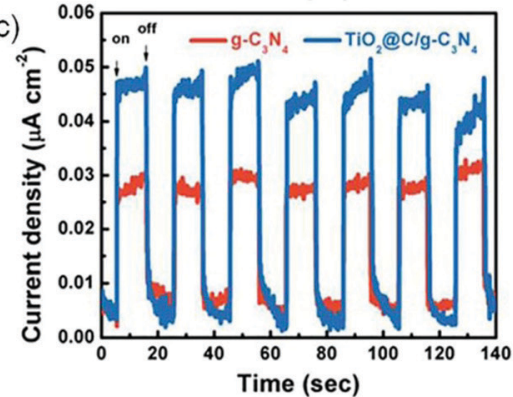

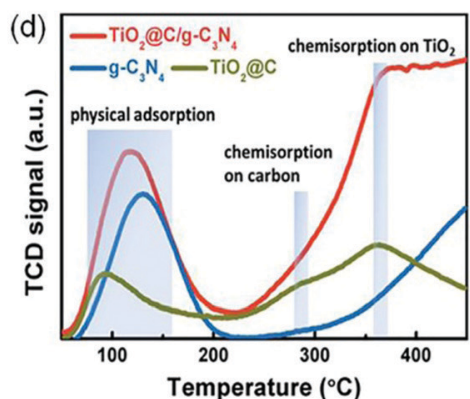

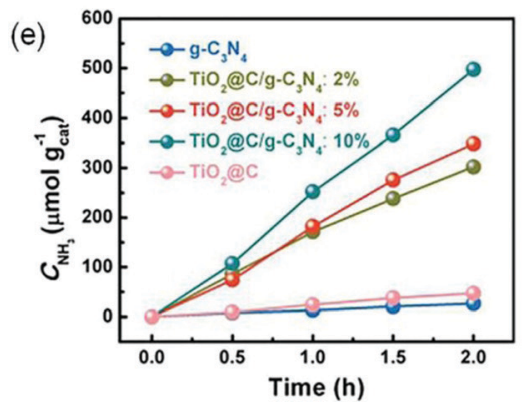

Fig. 12 (a) $\mathrm{SEM}$ image of $\mathrm{TiO}_{2} @ \mathrm{aC} / \mathrm{g}-\mathrm{C}_{3} \mathrm{~N}_{4}$. (b) PL spectra of $\mathrm{TiO}_{2} @ \mathrm{aC} / \mathrm{g}-\mathrm{C}_{3} \mathrm{~N}_{4}$ and g- $\mathrm{C}_{3} \mathrm{~N}_{4}$. (c) Transient photocurrent responses of TiO $2 \mathrm{QC} / \mathrm{g}-\mathrm{C}_{3} \mathrm{~N}_{4}$ and $\mathrm{g}-\mathrm{C}_{3} \mathrm{~N}_{4}$. (d) The $\mathrm{N}_{2}$-TPD profiles. (e) The yield of produced $\mathrm{NH}_{3}$ under visible light irradiation $(\lambda>420 \mathrm{~nm})$. Reproduced from ref. 112 with permission from The Royal Society of Chemistry. 
for the adsorption and activation of $\mathrm{N}_{2}$ in $\mathrm{TiO}_{2} @ \mathrm{C} / \mathrm{g}-\mathrm{C}_{3} \mathrm{~N}_{4}$ (Fig. 12d). As a result, a superior performance over the optimal $\mathrm{TiO}_{2} @ \mathrm{C} / \mathrm{g}-\mathrm{C}_{3} \mathrm{~N}_{4}: 10$ (10 represents the molar ratio of starting materials of melamine $/ \mathrm{Ti}_{3} \mathrm{C}_{2}$ ) was realized with an $\mathrm{NH}_{3}$ production yield of $250.6 \mu \mathrm{mol} \mathrm{g}{ }^{-1} \mathrm{~h}^{-1}$ using methanol as a scavenger under visible light irradiation (Fig. 12e). Recently, a p-n junction of $\mathrm{Bi}_{2} \mathrm{MoO}_{6} / \mathrm{OV}$-BiOBr combining n-type $\mathrm{Bi}_{2} \mathrm{MoO}_{6}$ nanorods with OVrich p-type $\mathrm{BiOBr}$ nanosheets displayed efficient charge separation and a broad range of light absorption. ${ }^{116}$ Moreover, OVs on the surface of $\mathrm{Bi}_{2} \mathrm{MoO}_{6} / \mathrm{OV}$-BiOBr were beneficial for the chemisorption and activation of $\mathrm{N}_{2}$, and the nanosheet morphology could provide a large specific surface area with more active sites for photoreduction of $\mathrm{N}_{2}$. Benefiting from these advantages, the optimal $\mathrm{Bi}_{2} \mathrm{MoO}_{6} / \mathrm{OV}-\mathrm{BiOBr}$ exhibited a photocatalytic yield of $\mathrm{NH}_{3}$ of $81.0 \mu \mathrm{mol} \mathrm{g}{ }^{-1} \mathrm{~h}^{-1}$ under visible light illumination in pure water, and no $\mathrm{O}_{2}$ production was reported here.

\section{Conclusion and perspectives}

The photosynthesis of $\mathrm{NH}_{3}$ is an energy-saving process and has high potential for substantial contribution to economic and social sustainability. Compared with the traditional HaberBosch process and the emerging electrochemical process, photocatalytic $\mathrm{NH}_{3}$ synthesis exhibits very low efficiency, mostly at micromoles $\mathrm{g}^{-1} \mathrm{~h}^{-1}$ if using water as the electron donor, and thus is far from practical application. However as photocatalysis can be driven by abundant solar energy, it is a carbon-free process. Besides, it requires a solar energy input of 208.3 $\mathrm{MJ} \mathrm{kg}^{-1}-\mathrm{NH}_{3}$ compared with that of $339.1 \mathrm{MJ} \mathrm{kg}^{-1}-\mathrm{NH}_{3}$ in the electrochemical process. ${ }^{117}$ Thus, it has strong potential to achieve $\mathrm{NH}_{3}$ synthesis with affordable costs in particular taking into account the sustainability and the trend of decarbonatization in the industry. However there is a long way to go in order to realize this potential.

This review does not detail different photocatalysts as there are a few reviews on them, but analyses surface engineering and interface engineering in photocatalysts developed recently for the photocatalytic $\mathrm{NH}_{3}$ synthesis, involving oxygen vacancies, nitrogen vacancies, carbon vacancies, sulfur vacancies, metal and non-metal doping, facet modulation, nanostructure engineering, and heterostructure construction such as loading with cocatalysts and modification with other semiconductors, together with the mechanism of $\mathrm{N}_{2}$ reduction reaction and the reliable means for the detection of $\mathrm{NH}_{3}$.

Great progress has been made in the study of photoreduction of $\mathrm{N}_{2}$ to $\mathrm{NH}_{3}$ as briefed in Fig. 13, but there are still many problems, some of which are very critical. First, the majority of the studies so far reported use an efficient and expensive hole scavenger to get long lived photoelectrons for $\mathrm{N}_{2}$ reduction. However, a practical pathway should use water as the only electron donor and to provide protons for the photosynthesis of $\mathrm{NH}_{3}$, as such $\mathrm{NH}_{3}$ synthesis by photocatalysis is scientifically meaningful and economically sustainable. The slow progress linked to this profile is mainly due to the very limited understanding in this field. Second, there are few reports specifying

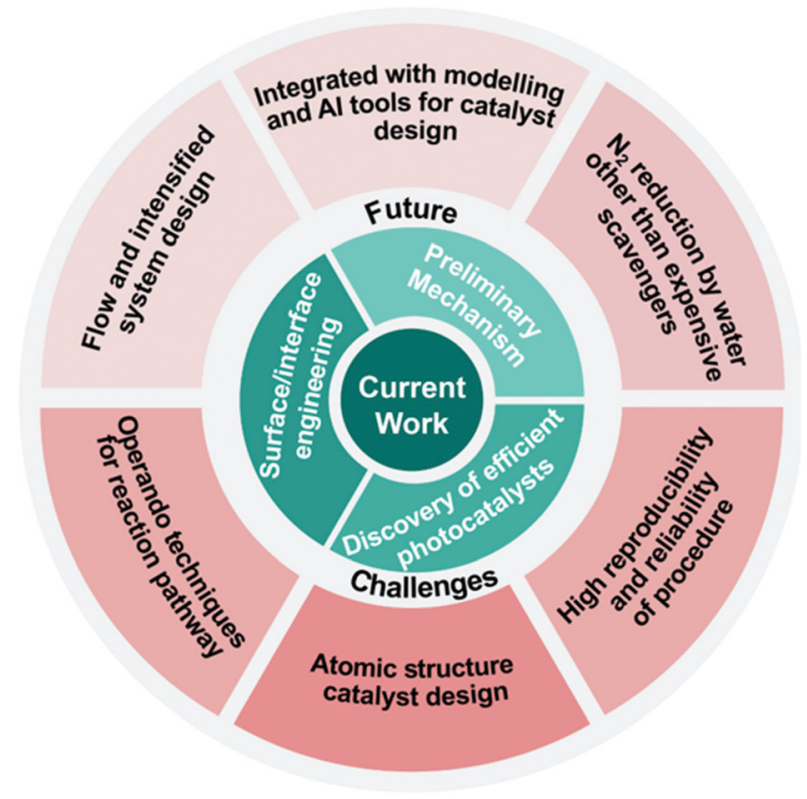

Fig. 13 Current work and future challenges highlighted in this review.

the stoichiometric chemistry in the photochemical process. Without the stoichiometric $\mathrm{O}_{2}$ gas produced, such $\mathrm{N}_{2}$ reduction to $\mathrm{NH}_{3}$ is questionable. Furthermore, as $\mathrm{O}_{2}$ could be leaked from air, the isotopic measurement of $\mathrm{O}_{2}$ production is necessary. In addition, since $\mathrm{H}_{2}$ production would compete with $\mathrm{NH}_{3}$ synthesis, a comprehensive study should detail the selectivity between the two products and discuss the strategy to favor $\mathrm{NH}_{3}$ synthesis. In addition an experimental error bar is very crucial for such $\mathrm{NH}_{3}$ synthesis in order to improve the confidence as almost all studies were carried out in a batch reactor with quite a small amount of $\mathrm{NH}_{3}$ produced, which was quite easy to be interfered by occasional factors.

Apart from the issues mentioned above, photocatalytic $\mathrm{NH}_{3}$ synthesis also faces a few tough challenges. The majority of the studies show that the $\mathrm{NH}_{4}$ amount produced is less than $10 \mu \mathrm{mol} \mathrm{h}{ }^{-1}$ (instead of the unit of $\mu \mathrm{mol} \mathrm{g}{ }^{-1} \mathrm{~h}^{-1}$ ) when using water as the electron donor and 10 times enhancement is achieved when using an organic hole scavenger. This amount is lower than the $\mathrm{NH}_{3}$ amount in the river water which is about $0.01 \mathrm{mM} .{ }^{118}$ Besides, since the $\mathrm{NH}_{3}$ yield is extremely easy to be interfered by environmental impurities and different detection agents, it is difficult to obtain reliable and reproducible results by just one analysis method. ${ }^{119}$ At least two methods have to be applied to prove the consistent results and to improve the reproducibility of the process.

The surface states of a catalyst can dominate its performance, such as surface defects. However, to quantify the correlation of the surface defects with the catalytic activity is very challenging at present. This should be undertaken in the future in order to distinctly guide catalyst surface state control. Furthermore the cocatalyst is another key factor to control both charge separation and catalytic performance. The majority of the cocatalysts reported are either large particles or nanosized particles. Single atomic catalysis is an emerging area, which 
presents unprecedented activity in some chemical processes. Such unique reaction sites should be introduced to ammonia synthesis with in-depth exploration of their intrinsic function. In addition, a junction structure is widely accepted to help charge separation, which was also reviewed in the previous session. The new design strategy should be applied to construct new junctions, such as polymer and oxide junctions as the former has likely better $\mathrm{N}_{2}$ adsorption due to the potential hydrogen bond between the organic polymer and $\mathrm{N}_{2}$ while the latter has a very positive VB for water oxidation.

The modified catalyst discovery is very informative. Equally, the mechanism and reaction pathways for photocatalytic $\mathrm{N}_{2}$ fixation are scarce and much less investigated, forming an obstacle for the rational design of highly efficient photocatalysts. Currently, most of the given reaction mechanisms and reaction pathways were based on assumptions and lacked solid evidence. Thus, the current catalyst development is more or less a try-and-error process. Some advanced operando technologies in functional characterization should be applied to comprehensively study the reaction pathway such as by time resolved spectroscopy to investigate charge carrier dynamics, and by transient and stationary spectroscopy to observe the reaction intermediates.

In addition, it is of paramount importance to design a flow reaction system for $\mathrm{N}_{2}$ photoreduction. The current reaction system is composed of a batch reactor, which is far from the requirement of the industry. The typical batch reactor is easy to be constructed; however, its efficiency is severely restricted by the low mass transfer between $\mathrm{N}_{2}$ gas and aqueous solution due to the low solubility of $\mathrm{N}_{2}$, thereby resulting in a low photocatalytic efficiency. Besides, $\mathrm{NH}_{3}$ accumulated in the batch reactor during the reaction process will be overoxidized to other byproducts, thereby resulting in a low conversion rate and even bad selectivity. Moreover, based on the industrial viewpoint, it is quite costly to separate the photocatalysts from reactants and products in a batch system. Thus, an intensified flow reactor such as using $\mathrm{N}_{2}$ and water vapor as reactants should be explored to overcome these drawbacks and to meet the industrial requirement in the future, underlining the crucial collaborations between chemical scientists and experts in reaction engineering.

Last, modelling will be expected to combine with experimental results to figure out the reaction mechanism and speed up the rational design of highly efficient photocatalysts. Meanwhile, the big data, together with artificial intelligence (AI) and machine learning (ML), will be a future key research area to improve the reproducibility of the catalyst synthesis, to facilitate catalyst screening, to enhance the experimental safety and to save the experimental costs and time.

Overall, this research field is at an infant state and all these areas should be enhanced in order to improve the $\mathrm{NH}_{3}$ synthesis rate with high reproducibility and reliability of the procedure.

\section{Conflicts of interest}

There are no conflicts to declare.

\section{Acknowledgements}

Q. H. is thankful for the NSFC grants (21575014 and 21905025), Beijing Natural Science Foundation (2184122), the Fundamental Research Funds for the Central Universities (2018CX01017), Beijing Institute of Technology Research Fund Program for Young Scholars, and Analysis \& Testing Center at Beijing Institute of Technology. H.M.J is thankful for the UCL Dean's prize and China CSC scholarship. All authors are thankful for financial support from UK EPSRC (EP/N009533/1), Royal Society-Newton Advanced Fellowship Grant (NA170422) and the Leverhulme Trust (RPG-2017-122).

\section{References}

1 V. Smil, Nature, 1999, 400, 415.

2 H. P. Jia and E. A. Quadrelli, Chem. Soc. Rev., 2014, 43, 547-564.

3 K. Ithisuphalap, H. Zhang, L. Guo, Q. Yang, H. Yang and G. Wu, Small Methods, 2019, 3, 1800352.

4 G. Ertl, Angew. Chem., Int. Ed., 2008, 47, 3524-3535.

5 R. Schlögl, Angew. Chem., Int. Ed., 2003, 42, 2004-2008.

6 C. Mao, J. Wang, Y. Zou, H. Li, G. Zhan, J. Li, J. Zhao and L. Zhang, Green Chem., 2019, 21, 2852-2867.

7 P. Wang, F. Chang, W. Gao, J. Guo, G. Wu, T. He and P. Chen, Nat. Chem., 2017, 9, 64-70.

8 N. Cherkasov, A. O. Ibhadon and P. Fitzpatrick, Chem. Eng. Process., 2015, 90, 24-33.

9 G. Duan, Y. Chen, Y. Tang, K. A. M. Gasem, P. Wan, D. Ding and M. Fan, Prog. Energy Combust. Sci., 2020, 81, 100860.

10 R. Zhao, H. Xie, L. Chang, X. Zhang, X. Zhu, X. Tong, T. Wang, Y. Luo, P. Wei, Z. Wang and X. Sun, EnergyChem, 2019, 1, 100011.

11 S. C. Sun, X. Y. Zhang, X. L. Liu, L. Pan, X. W. Zhang and J. J. Zou, Acta Phys.-Chim. Sin., 2020, 36, 1905007.

12 Y. Zhao, R. Shi, X. Bian, C. Zhou, Y. Zhao, S. Zhang, F. Wu, G. I. N. Waterhouse, L. Z. Wu, C. H. Tung and T. Zhang, Adv. Sci., 2019, 6, 1802109.

13 Y. Huang, N. Zhang, Z. Wu and X. Xie, J. Mater. Chem. A, 2020, 8, 4978-4995.

14 A. J. Medford and M. C. Hatzell, ACS Catal., 2017, 7, 2624-2643.

15 C. Guo, J. Ran, A. Vasileff and S. Z. Qiao, Energy Environ. Sci., 2018, 11, 45-56.

16 Y. J. Zhang, F. X. Mao, L. J. Wang, H. Y. Yuan, P. F. Liu and H. G. Yang, Sol. RRL, 2020, 4, 1900438.

17 G. N. Schrauzer and T. D. Guth, J. Am. Chem. Soc., 1977, 99, 7189-7193.

18 M. Cheng, C. Xiao and Y. Xie, J. Mater. Chem. A, 2019, 7, 19616-19633.

19 D. Yan, H. Li, C. Chen, Y. Zou and S. Wang, Small Methods, 2019, 3, 1800331.

20 S. Zhang, Y. Zhao, R. Shi, C. Zhou, G. I. N. Waterhouse, L. Z. Wu, C. H. Tung and T. Zhang, Adv. Energy Mater., 2020, 10, 1901973. 
21 Y. Zhao, R. Shi, X. Bian, C. Zhou, Y. Zhao, S. Zhang, F. Wu, G. I. N. Waterhouse, L. Z. Wu, C. H. Tung and T. Zhang, Adv. Sci., 2019, 6, 1802109.

22 X. Chen, N. Li, Z. Kong, W. J. Ong and X. Zhao, Mater. Horiz., 2018, 5, 9-27.

23 X. Xue, R. Chen, H. Chen, Y. Hu, Q. Ding, Z. Liu, L. Ma, G. Zhu, W. Zhang, Q. Yu, J. Liu, J. Ma and Z. Jin, Nano Lett., 2018, 18, 7372-7377.

24 X. Y. Xie, P. Xiao, W.-H. Fang, G. Cui and W. Thiel, ACS Catal., 2019, 9, 9178-9187.

25 A. J. Medford and M. C. Hatzell, ACS Catal., 2017, 7, 2624-2643.

26 J. Li, H. Li, G. Zhan and L. Zhang, Acc. Chem. Res., 2017, 50, 112-121.

27 M. A. Shipman and M. D. Symes, Catal. Today, 2017, 286, 57-68.

28 C. Ling, Y. Zhang, Q. Li, X. Bai, L. Shi and J. Wang, J. Am. Chem. Soc., 2019, 141, 18264-18270.

29 X. Gao, Y. Wen, D. Qu, L. An, S. Luan, W. Jiang, X. Zong, X. Liu and Z. Sun, ACS Sustainable Chem. Eng., 2018, 6, 5342-5348.

30 A. Banerjee, B. D. Yuhas, E. A. Margulies, Y. Zhang, Y. Shim, M. R. Wasielewski and M. G. Kanatzidis, J. Am. Chem. Soc., 2015, 137, 2030-2034.

31 H. Li, J. Shang, Z. Ai and L. Zhang, J. Am. Chem. Soc., 2015, 137, 6393-6399.

32 H. Hirakawa, M. Hashimoto, Y. Shiraishi and T. Hirai, J. Am. Chem. Soc., 2017, 139, 10929-10936.

33 C. Li, T. Wang, Z. J. Zhao, W. Yang, J.-F. Li, A. Li, Z. Yang, G. A. Ozin and J. Gong, Angew. Chem., Int. Ed., 2018, 57, 5278-5282.

34 Y. Zhao, X. Jia, G. I. N. Waterhouse, L.-Z. Wu, C. H. Tung, D. O'Hare and T. Zhang, Adv. Energy Mater., 2016, 6, 1501974.

35 S. Zhang, Y. Zhao, R. Shi, C. Zhou, G. I. N. Waterhouse, L. Z. Wu, C. H. Tung and T. Zhang, Adv. Energy Mater., 2020, 10, 1901973.

36 Y. Zhao, Y. Zhao, G. I. N. Waterhouse, L. Zheng, X. Cao, F. Teng, L. Z. Wu, C. H. Tung, D. O'Hare and T. Zhang, Adv. Mater., 2017, 29, 1703828.

37 L. Ye, L. Zan, L. Tian, T. Peng and J. Zhang, Chem. Commun., 2011, 47, 6951-6953.

38 H. Li and L. Zhang, Nanoscale, 2014, 6, 7805-7810.

39 J. Chen, T. Ding, J. Cai, Y. Wang, M. Wu, H. Zhang, W. Zhao, Y. Tian, X. Wang and X. Li, Appl. Surf. Sci., 2018, 453, 101-109.

40 Y. Wang, J. Cai, M. Wu, J. Chen, W. Zhao, Y. Tian, T. Ding, J. Zhang, Z. Jiang and X. Li, Appl. Catal., B, 2018, 239, 398-407.

41 Y. Huang, Y. Yu, Y. Yu and B. Zhang, Sol. RRL, 2020, 4, 2000037.

42 Q. Han, B. Wang, J. Gao, Z. Cheng, Y. Zhao, Z. Zhang and L. Qu, ACS Nano, 2016, 10, 2745-2751.

43 Q. Han, Z. Cheng, B. Wang, H. Zhang and L. Qu, ACS Nano, 2018, 12, 5221-5227.

44 H. Ou, L. Lin, Y. Zheng, P. Yang, Y. Fang and X. Wang, Adv. Mater., 2017, 29, 1700008.
45 Q. Han, B. Wang, J. Gao and L. Qu, Angew. Chem., Int. Ed., 2016, 55, 10849-10853.

46 Q. Han, B. Wang, Y. Zhao, C. Hu and L. Qu, Angew. Chem., Int. Ed., 2015, 54, 11433-11437.

47 Y. Liang, F. Liu, Y. Deng, Q. Zhou, Z. Cheng, P. Zhang, Y. Xiao, L. Lv, H. Liang, Q. Han, H. Shao and L. Qu, Small, 2018, 14, 1801916.

48 Q. Han, C. Hu, F. Zhao, Z. Zhang, N. Chen and L. Qu, J. Mater. Chem. A, 2015, 3, 4612-4619.

49 L. Chen, Y. Wang, C. Wu, G. Yu, Y. Yin, C. Su, J. Xie, Q. Han and L. Qu, Nanoscale, 2020, 12, 13484-13490.

50 Q. Han, N. Chen, J. Zhang and L. Qu, Mater. Horiz., 2017, 4, 832-850.

51 B. He, M. Feng, X. Chen and J. Sun, Green Energy Environ., 2020, DOI: 10.1016/j.gee.2020.07.011.

52 Y. Wang and S. Shen, Acta Phys.-Chim. Sin., 2020, 36, 1905080.

53 W. J. Ong, L. K. Putri and A. R. Mohamed, Chem. - Eur. J., 2020, 26, 9710-9748.

54 G. Dong, W. Ho and C. Wang, J. Mater. Chem. A, 2015, 3, 23435-23441.

55 W. Wang, H. Zhang, S. Zhang, Y. Liu, G. Wang, C. Sun and H. Zhao, Angew. Chem., Int. Ed., 2019, 11, 16644-16650.

56 S. Cao, B. Fan, Y. Feng, H. Chen, F. Jiang and X. Wang, Chem. Eng. J., 2018, 353, 147-156.

57 Y. Cao, S. Hu, F. Li, Z. Fan, J. Bai, G. Lu and Q. Wang, RSC Adv., 2016, 6, 49862-49867.

58 V. Kumaravel, S. Mathew, J. Bartlett and S. C. Pillai, Appl. Catal., B, 2019, 244, 1021-1064.

59 Z. Shayegan, C. S. Lee and F. Haghighat, Chem. Eng. J., 2018, 334, 2408-2439.

60 J. J. Carey and M. Nolan, J. Mater. Chem. A, 2017, 5, 15613-15630.

61 Y. Shi, Y. Zhou, D. R. Yang, W. X. Xu, C. Wang, F. B. Wang, J. J. Xu, X.-H. Xia and H. Y. Chen, J. Am. Chem. Soc., 2017, 139, 15479-15485.

62 E. Paek, A. J. Pak and G. S. Hwang, ACS Appl. Mater. Interfaces, 2014, 6, 12168-12176.

63 J. Zhang, Y. Liu, C. Sun, P. Xi, S. Peng, D. Gao and D. Xue, ACS Energy Lett., 2018, 3, 779-786.

64 W. Zhao, J. Zhang, X. Zhu, M. Zhang, J. Tang, M. Tan and Y. Wang, Appl. Catal., B, 2014, 144, 468-477.

65 J. Luo, X. Bai, Q. Li, X. Yu, C. Li, Z. Wang, W. Wu, Y. Liang, Z. Zhao and H. Liu, Nano Energy, 2019, 66, 104187.

66 N. Zhang, L. Li, Q. Shao, T. Zhu, X. Huang and X. Xiao, ACS Appl. Energy Mater., 2019, 2, 8394-8398.

67 N. Zhang, A. Jalil, D. Wu, S. Chen, Y. Liu, C. Gao, W. Ye, Z. Qi, H. Ju, C. Wang, X. Wu, L. Song, J. Zhu and Y. Xiong, J. Am. Chem. Soc., 2018, 140, 9434-9443.

68 Y. Zhao, Y. Zhao, R. Shi, B. Wang, G. I. N. Waterhouse, L. Z. Wu, C. H. Tung and T. Zhang, Adv. Mater., 2019, 31, 1806482.

69 Y. Bi, S. Ouyang, N. Umezawa, J. Cao and J. Ye, J. Am. Chem. Soc., 2011, 133, 6490-6492.

70 P. V. Kamat, Acc. Chem. Res., 2017, 50, 527-531.

71 Q. Hao, C. Liu, G. Jia, Y. Wang, H. Arandiyan, W. Wei and B. J. Ni, Mater. Horiz., 2020, 7, 1014-1029. 
72 Z. Li, Z. Gao, B. Li, L. Zhang, R. Fu, Y. Li, X. Mu and L. Li, Appl. Catal., B, 2020, 262, 118276.

73 W. Wang, H. Zhou, Y. Liu, S. Zhang, Y. Zhang, G. Wang, H. Zhang and H. Zhao, Small, 2020, 16, 1906880.

74 M. Setvin, X. Hao, B. Daniel, J. Pavelec, Z. Novotny, G. S. Parkinson, M. Schmid, G. Kresse, C. Franchini and U. Diebold, Angew. Chem., Int. Ed., 2014, 53, 4714-4716.

75 J. Bai, B. Lu, Q. Han, Q. Li and L. Qu, ACS Appl. Mater. Interfaces, 2018, 10, 38066-38072.

76 G. Liu, J. C. Yu, G. Q. Lu and H. M. Cheng, Chem. Commun., 2011, 47, 6763-6783.

77 Y. Bai, L. Ye, T. Chen, L. Wang, X. Shi, X. Zhang and D. Chen, ACS Appl. Mater. Interfaces, 2016, 8, 27661-27668.

78 H. Li, J. Shang, J. Shi, K. Zhao and L. Zhang, Nanoscale, 2016, 8, 1986-1993.

79 Q. Han, Z. Cheng, J. Gao, Y. Zhao, Z. Zhang, L. Dai and L. Qu, Adv. Funct. Mater., 2017, 27, 1606352.

80 Y. O. Wang, F. Silveri, M. K. Bayazit, Q. S. Ruan, Y. M. Li, J. J. Xie, C. R. A. Catlow and J. W. Tang, Adv. Energy Mater., 2018, 8, 1801084.

81 Q. Han, F. Zhao, C. Hu, L. Lv, Z. Zhang, N. Chen and L. Qu, Nano Res., 2015, 8, 1718-1728.

82 J. Bai, Q. Han, Z. Cheng and L. Qu, Chem. - Asian J., 2018, 13, 3160-3164.

83 L. W. Chen, X. T. Ding, J. F. Zeng, C. B. Wu, Q. Han and L. T. Qu, Sci. Bull., 2019, 54, 718-722.

84 Y. Wang, R. Xu, L. Chen, C. Wu, L. Qiu, C. D. Windle, Q. Han and L. Qu, ACS Appl. Mater. Interfaces, 2020, 12, 8547-8554.

85 G. Zhang, C. D. Sewell, P. Zhang, H. Mi and Z. Lin, Nano Energy, 2020, 71, 104645.

86 S. Sun, Q. An, W. Wang, L. Zhang, J. Liu and W. A. Goddard III, J. Mater. Chem. A, 2017, 5, 201-209.

87 S. Wang, X. Hai, X. Ding, K. Chang, Y. Xiang, X. Meng, Z. Yang, H. Chen and J. Ye, Adv. Mater., 2017, 29, 1701774.

88 S. Bian, M. Wen, J. Wang, N. Yang, P. K. Chu and X. F. Yu, J. Phys. Chem. Lett., 2020, 11, 1052-1058.

89 L. Lin, Q. Zhu, A. Cheng and L. Ma, Catal. Sci. Technol., 2020, 10, 4119-4125.

90 J. Ran, J. Zhang, J. Yu, M. Jaroniec and S. Z. Qiao, Chem. Soc. Rev., 2014, 43, 7787-7812.

91 R. Li, H. Han, F. Zhang, D. Wang and C. Li, Energy Environ. Sci., 2014, 7, 1369-1376.

92 G. Zhang, Z. A. Lan and X. Wang, Chem. Sci., 2017, 8, 5261-5274.

93 K. T. Ranjit, T. K. Varadarajan and B. Viswanathan, J. Photochem. Photobiol., A, 1996, 96, 181-185.

94 Q. Zhang and J. Guan, Sol. RRL, 2020, 4, 2000283.

95 S. Liu, Y. Wang, S. Wang, M. You, S. Hong, T. S. Wu, Y. L. Soo, Z. Zhao, G. Jiang, Q. Jieshan, B. Wang and Z. Sun, ACS Sustainable Chem. Eng., 2019, 7, 6813-6820.

96 J. Yang, Y. Guo, R. Jiang, F. Qin, H. Zhang, W. Lu, J. Wang and J. C. Yu, J. Am. Chem. Soc., 2018, 140, 8497-8508.
97 R. Wang, T. Xie, Z. Sun, T. Pu, W. Li and J.-P. Ao, RSC Adv., 2017, 7, 51687-51694.

98 X. W. Guo, S. M. Chen, H. J. Wang, Z. M. Zhang, H. Lin, L. Song and T. B. Lu, J. Mater. Chem. A, 2019, 7, 19831-19837.

99 S. Chang and X. Xu, Inorg. Chem. Front., 2020, 7, 620-624.

100 J. Wang, C. Hua, X. Dong, Y. Wang and N. Zheng, Sustainable Energy Fuels, 2020, 4, 1855-1862.

101 J. J. Xie, R. Jin, A. Li, Q. Ruan, Y. Deng, Y. Zhang, S. Yao, G. Sankar, D. Ma and J. W. Tang, Nat. Catal., 2018, 11, 889-896.

102 J. Yang, D. Wang, H. Han and C. Li, Acc. Chem. Res., 2013, 46, 1900-1909.

103 Y. Liao, J. Qian, G. Xie, Q. Han, W. Dang, Y. Wang, L. Lv, S. Zhao, L. Luo, W. Zhang, H.-Y. Jiang and J. Tang, Appl. Catal., B, 2020, 273, 119054.

104 C. M. Janet, S. Navaladian, B. Viswanathan, T. K. Varadarajan and R. P. Viswanath, J. Phys. Chem. C, 2010, 114, 2622-2632.

105 B. Sun, Z. Liang, Y. Qian, X. Xu, Y. Han and J. Tian, ACS Appl. Mater. Interfaces, 2020, 12, 7257-7269.

106 X. Gao, L. An, D. Qu, W. Jiang, Y. Chai, S. Sun, X. Liu and Z. Sun, Sci. Bull., 2019, 64, 918-925.

107 J. Li, P. Liu, Y. Tang, H. Huang, H. Cui, D. Mei and C. Zhong, ACS Catal., 2020, 10, 2431-2442.

108 H. Wang, X. Li, Q. Ruan and J. Tang, Nanoscale, 2020, 12, 12329-12335.

109 S. X. Wang, H. Maimaiti, B. Xu, Y. Guo, P. S. Zhai and H. Z. Zhang, J. Phys. Chem. C, 2019, 123, 31119-31129.

110 Y. Lu, Y. Yang, T. Zhang, Z. Ge, H. Chang, P. Xiao, Y. Xie, L. Hua, Q. Li, H. Li, B. Ma, N. Guan, Y. Ma and Y. Chen, ACS Nano, 2016, 10, 10507-10515.

111 E. Vesali-Kermani, A. Habibi-Yangjeh, H. DiarmandKhalilabad and S. Ghosh, J. Colloid Interface Sci., 2020, 563, 81-91.

112 Q. Liu, L. Ai and J. Jiang, J. Mater. Chem. A, 2018, 6, 4102-4110.

113 H. Mou, J. Wang, D. Zhang, D. Yu, W. Chen, D. Wang and T. Mu, J. Mater. Chem. A, 2019, 7, 5719-5725.

114 W. J. Ong and K. P. Y. Shak, Sol. RRL, 2020, 4, 2000132.

115 W. Zhang, A. R. Mohamed and W. J. Ong, Angew. Chem., Int. Ed., 2020, DOI: 10.1002/anie.201914925.

116 X. Xue, R. Chen, C. Yan, Y. Hu, W. Zhang, S. Yang, L. Ma, G. Zhu and Z. Jin, Nanoscale, 2019, 11, 10439-10445.

117 L. Wang, M. Xia, H. Wang, K. Huang, C. Qian, C. T. Maravelias and G. A. Ozin, Joule, 2018, 2, 1055-1074.

118 S. Gandaseca, N. Rosli, J. Ngayop and C. Arianto, Am. J. Environ. Sci., 2011, 7, 269-275.

119 S. Z. Andersen, V. Čolić, S. Yang, J. A. Schwalbe, A. C. Nielander, J. M. McEnaney, K. Enemark-Rasmussen, J. G. Baker, A. R. Singh, B. A. Rohr, M. J. Statt, S. J. Blair, S. Mezzavilla, J. Kibsgaard, P. C. K. Vesborg, M. Cargnello, S. F. Bent, T. F. Jaramillo, I. E. L. Stephens, J. K. Nørskov and I. Chorkendorff, Nature, 2019, 570, 504-508. 